\title{
In Vivo Diffusion Tensor Imaging in Acute and Subacute Phases of Mild Traumatic Brain Injury in Rats
}

Isabel San Martín Molina, Raimo A. Salo, Ali Abdollahzadeh, Jussi Tohka, Olli Gröhn, and

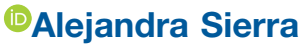

https://doi.org/10.1523/ENEURO.0476-19.2020

A.I. Virtanen Institute for Molecular Sciences, University of Eastern Finland, Kuopio FI-70211, Finland

\section{Visual Abstract}
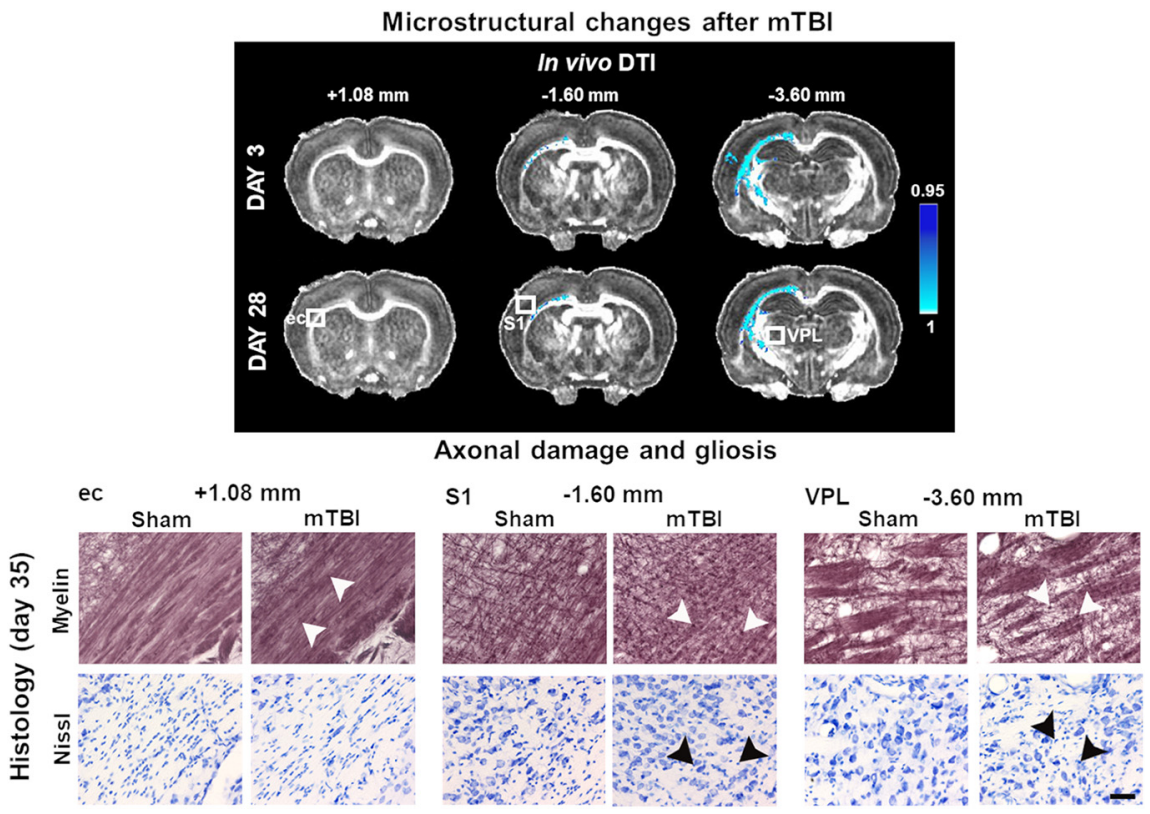

Mild traumatic brain injury (mTBI) is the most common form of TBI with $10-25 \%$ of the patients experiencing long-lasting symptoms. The potential of diffusion tensor imaging (DTI) for evaluating microstructural damage

\section{Significance Statement}

Mild traumatic brain injury (mTBI) is a major health problem worldwide with an unclear diagnosis. Using the lateral fluid percussion (LFP) injury model in rats, we induced mTBI to assess the potential of in vivo diffusion tensor imaging (DTI) for non-invasively detecting progressive microstructural tissue damage. To interpret the changes observed in DTI, we performed extensive quantitative histologic assessment of the tissue microstructure. From the acute to subacute phases after $\mathrm{mTBI}$, in vivo DTI detected progressive microstructural tissue alterations in the white and gray matter associated with axonal damage and gliosis. Although in vivo DTI failed to detect secondary tissue damage far from the primary lesion, these findings provide new insights for detecting mild tissue damage using in vivo DTI. 
after TBI is widely recognized, but the interpretation of DTI changes and their relationship with the underlying tissue damage is unclear. We studied how both axonal damage and gliosis contribute to DTI alterations after mTBI. We induced mTBI using the lateral fluid percussion (LFP) injury model in adult male Sprague Dawley rats and scanned them at 3 and $28 \mathrm{~d}$ post-mTBI. To characterize the DTI findings in the tissue, we assessed the histology by performing structure tensor (ST)-based analysis and cell counting on myelin-stained and Nissl-stained sections, respectively. In particular, we studied the contribution of two tissue components, myelinated axons and cellularity, to the DTI changes. Fractional anisotropy (FA), mean diffusivity (MD), and axial diffusivity (AD) were decreased in both white and gray matter areas in the acute phase post-mTBI, mainly at the primary lesion site. In the subacute phase, FA and AD were decreased in the white matter, external capsule, corpus callosum, and internal capsule. Our quantitative histologic assessment revealed axonal damage and gliosis throughout the brain in both white and gray matter, consistent with the FA and $A D$ changes. Our findings suggest that the usefulness of in vivo DTI is limited in its detection of secondary damage distal to the primary lesion, while at the lesion site, DTI detected progressive microstructural damage in the white and gray matter after mTBI.

Key words: axonal damage; cell counting; diffusion tensor imaging; inflammation; mild traumatic brain injury; secondary damage; structure tensor

\section{Introduction}

Mild traumatic brain injury (mTBI) is the most common form of $\mathrm{TBI}$, affecting 42 million people worldwide per year (Gardner and Yaffe, 2015). Clinically, mTBI is defined as a mild insult to the head causing a brief ( $<30 \mathrm{~min})$ period of unconsciousness and/or confusion and disorientation (Arciniegas et al., 2005; Mclnnes et al., 2017). Because of the absence of physical signs of severe injury, most mTBI patients are discharged without follow-up (Mayer et al., 2017). However, $\sim 10-25 \%$ of the patients with $\mathrm{mTBI}$ suffer long-term consequences such as depression, attention or memory problems, and sleep or mood disorders, which still persist one year after the injury (Grandhi et al., 2017).

Computed tomography (CT) and MRI are the goldstandard techniques for clinically assessing tissue damage after TBI (Kim and Gean, 2011). CT is commonly available and used in the acute phase of TBI for initial assessment of the damage severity (Mutch et al., 2016). Currently, MRI methods such as T1- and T2-weighted, susceptibility-weighted imaging, and diffusion-weighted imaging are used in routine clinical practice in both acute and chronic phases of head injury (Amyot et al., 2015). These MRI methods are sensitive to macroscopic damage such as parenchymal bleeding, edema, and

Received November 10, 2019; accepted May 11, 2020; First published May $18,2020$.

The authors declare no competing financial interests.

Author contributions: I.S.M.M., J.T., O.G., and A.S. designed research; I.S.M.M. performed research; I.S.M.M., R.A.S., A.A., and A.S. analyzed data; I.S.M.M., R.A.S., A.A., J.T., O.G., and A.S. wrote the paper.

This work was supported by the Academy of Finland Grants 275453 and 312686.

Acknowledgements: We thank Maarit Pulkkinen for her assistance in animal handling and histologic procedures and Karthik Chary for his assistance with the in vivo MRI data collection.

Correspondence should be addressed to Alejandra Sierra at alejandra. sierralopez@uef.fi.

https://doi.org/10.1523/ENEURO.0476-19.2020

Copyright @ 2020 San Martín Molina et al.

This is an open-access article distributed under the terms of the Creative Commons Attribution 4.0 International license, which permits unrestricted use, distribution and reproduction in any medium provided that the original work is properly attributed. penetrating injuries after moderate and severe TBI (Kim and Gean, 2011; Kinnunen et al., 2011). CT and conventional MRI approaches fail to detect widespread microscopic injuries such as axonal injury, however, which is commonly observed after mTBI in humans (Shenton et al., 2012) and characterized histologically in animals (Johnson et al., 2013).

Diffusion tensor imaging (DTI; Basser et al., 1994; Mori and Zhang, 2006) is widely used in experimental and clinical settings to investigate TBI due to its sensitivity to changes in the tissue microstructure (Pierpaoli et al., 1996; Sidaros et al., 2008; Laitinen et al., 2015; Sierra et al., 2015; Hutchinson et al., 2018; Tae et al., 2018). Several experimental in vivo DTI studies have characterized microstructural changes mainly in the acute phase of mTBI (Budde et al., 2013; Long et al., 2015; Wright et al., 2016; Li et al., 2016). The interpretation of changes in DTI metrics as an indicator of changes in the cellular level, however, is ambiguous. Only a few studies have combined DTI and histology, but only qualitative histologic analysis was applied with no direct link to the DTI metrics (Zhuo et al., 2012; Hylin et al., 2013; Singh et al., 2016; Tu et al., 2017; Herrera et al., 2017).

The aim of this study was to identify the spatial distribution of tissue damage throughout the rat brain in the acute and subacute phases of $\mathrm{mTBI}$ using in vivo MRI. As screening methods, we used in vivo voxel-wise and deformation-based morphometry analyses for DTI and T2weighted MRI data, respectively, to assess progressive microstructural alterations and local morphologic volumetric changes throughout the brain. To interpret DTI changes in the tissue microstructure, we applied a structure tensor (ST) analysis for myelin-stained sections and an automated cell counting method for Nissl-stained sections to examine the contribution of both myelinated axons and cellularity to the DTI parameters.

\section{Materials and Methods}

\section{Animals}

Adult male Sprague Dawley rats $(n=25,10$ weeks old, weight 300-450 g, Harlan Netherlands B.V.) were used in 
all the experiments. All the animals were housed individually in cages and maintained in a climate-controlled room (temperature $22 \pm 1^{\circ} \mathrm{C}$, air humidity $50-60 \%$ ) with a 12/ $12 \mathrm{~h} \mathrm{light/dark}$ cycle and an ad libitum diet. All the experimental procedures were approved by the Animal Ethics Committee of the Provincial Government of Southern Finland and conducted in accordance with the guidelines set by the European Union Directives 2010/63/EU.

\section{Animal model of mild TBI}

We induced $\mathrm{mTBI}$ using the lateral fluid percussion (LFP) injury model, as described previously (Kharatishvili et al., 2006). Briefly, rats were anesthetized by intraperitoneal injection $(6 \mathrm{ml} / \mathrm{kg})$ of a mixture of sodium pentobarbital $(58 \mathrm{mg} / \mathrm{kg})$, chloral hydrate $(60 \mathrm{mg} / \mathrm{kg})$, magnesium sulfate $(127.2 \mathrm{mg} / \mathrm{kg})$, propylene glycol $(42.8 \%)$, and absolute ethanol (11.6\%). We then performed a craniotomy ( $5 \mathrm{~mm}$ in diameter) between bregma and $\lambda$ on the left convexity (anterior edge $2.0 \mathrm{~mm}$ posterior to bregma; lateral edge adjacent to the left lateral ridge). LFP injury was induced $(n=13)$ by a transient fluid pulse impact (21-23 ms) against the exposed dura using a fluid percussion device (AmScien Instruments). The impact pressure was adjusted to induce a mild brain injury $(0.88 \pm 0.21 \mathrm{~atm})$. After the impact, we removed the animals from the fluid percussion device, and assessed the temporary absence of spontaneous breathing by measuring the apnea time. Additionally, we assessed the right-time reflex latency, presence of hematoma on the impact site and seizure post-mTBI. Sham-operated control animals $(n=12)$ underwent the same surgical procedures without the impact. Mild TBI animals recover same as the sham-operated animals after the impact, with no apnea, hematoma, or post-injury seizure. We also did not observe differences in right-time reflex latency in mTBI animals when comparing to sham-operated animals. The mortality during our experiments was $0 \%$.

\section{In vivo MRI}

All rats were scanned in vivo on days 3 and 28 after the injury under $1.0-1.5 \%$ isoflurane (in $70 \%$ nitrogen $/ 30 \%$ oxygen) anesthesia. We performed in vivo imaging using a horizontal 7 T Bruker PharmaScan MRI system with an actively decoupled quadrature volume transmitter coil and a quadrature receiver surface coil pair (Bruker Biospin). We monitored the breathing and temperature of the rats during the scans using a respiration pneumatic sensor and a rectal temperature probe, maintaining physiologic stability (breathing approximately at $60 \mathrm{bpm}$, temperature $\sim 37^{\circ} \mathrm{C}$ ) with a water circulation system (ThermoFisher Scientific). In vivo DTI data were acquired using three-dimensional (3D) spin echo-planar imaging sequences with respiratory gating and the following parameters: repetition time $=1000 \mathrm{~ms}$, echo time $=30 \mathrm{~ms}$, number of averages $=1$, field of view $=$ $21.4 \times 14.4 \times 15.6 \mathrm{~mm}^{3}$, bandwidth $=300 \mathrm{kHz}, \quad$ matrix size $=142 \times 96 \times 52$, b0 images $=4,60$ directions $(\delta=4 \mathrm{~ms}$, $\Delta=11 \mathrm{~ms}$, b-value $=2000 \mathrm{~s} / \mathrm{mm}^{2}$ ) with a resolution $=0.15 \times$ $0.15 \times 0.30 \mathrm{~mm}^{3}$, and a scan time of 2-3 h. We acquired T2weighted images using 2D fast spin-echo sequences with the following parameters: repetition time $=7800 \mathrm{~ms}$, echo time $=40 \mathrm{~ms}$, rapid acquisition relaxation enhancement factor $=8$, number of averages $=8$, field of view $=2.56 \times$ $2.56 \mathrm{~cm}^{2}$, matrix size $=170 \times 342$, resolution $=75 \times 150$ $\mu \mathrm{m}^{2}$, number of slices $=52$, slice thickness $=300 \mu \mathrm{m}$, and scan time $=22 \mathrm{~min}$.

\section{Statistical analyses of in vivo MRI}

In voxel-wise and deformation-based morphometry analyses, we used FSL randomize (Winkler et al., 2014) with 10,000 permutations and threshold-free cluster enhancement (TFCE; 3D-connectivity with standard parameters) to perform the statistical analyses. We used the permutation-based TFCE (Smith and Nichols, 2009) with the default parameters $(\mathrm{H}=2, \mathrm{E}=0.5, \mathrm{C}=6)$, which performs family-wise error (FWE) multiple comparison correction to the cluster-enhanced statistic values. For further discussion how to interpret TFCE results, we refer to Smith and Nichols (2009) and Noble et al. (2020).

\section{In vivo MRI data analysis}

For DTI analysis, we first converted the DTI data to Nifti format. Second, we preprocessed and performed all the analyses using tools in FMRIB Software Library (FSL 5.0.9; http:// www.fmrib.ox.ac.uk/fs//). Then, we performed simultaneous multiple correction for motion and eddy current distortions with the FSL eddy tool (Andersson and Sotiropoulos, 2016). For that, we used eddy unchanged parameters without the opposite phase encoding correction (topup). After eddy current corrections, we determined the diffusion tensors, and the respective eigenvalues $\left(\lambda_{1}, \lambda_{2}\right.$, and $\left.\lambda_{3}\right)$ of the diffusion tensors were calculated using FSL. We then generated fractional anisotropy (FA), axial diffusivity (AD), radial diffusivity (RD), and mean diffusivity (MD) maps (Pajevic and Pierpaoli, 1999). We also calculated linear (CL), planar (CP), and spherical (CS) anisotropy indices (Westin et al., 2002).

To determine the differences between the animal groups, we performed a voxel-wise statistical analysis of the whole brain between sham-operated and mTBI animals at both time-points. We created a study-specific FA template (the mean of five accurately registered sham-operated brain images). The template was then used in the affine and nonlinear [symmetric image normalization method (SyN); Avants et al., 2008] co-registration of sham-operated and mTBI brain images from both timepoints using Advanced Normalization Tools (ANTs; http:// stnava.github.io/ANTs/; Avants et al., 2011).

Additionally, based on the voxel-wise statistical analysis results on days 3 and 28 after injury, we used a region of interest (ROI) approach to correlate DTI data with the histologic analyses, selecting the same subgroup of animals used for the histologic procedures. In that subgroup, we manually outlined different brain areas at three different brain levels: two levels rostral to the lesion site, +1.08 and $-1.60 \mathrm{~mm}$ from bregma, and one at the level of the lesion, $-3.60 \mathrm{~mm}$ from bregma. We selected these three levels based on the absence or presence of major statistically significant differences in the DTI group analysis 


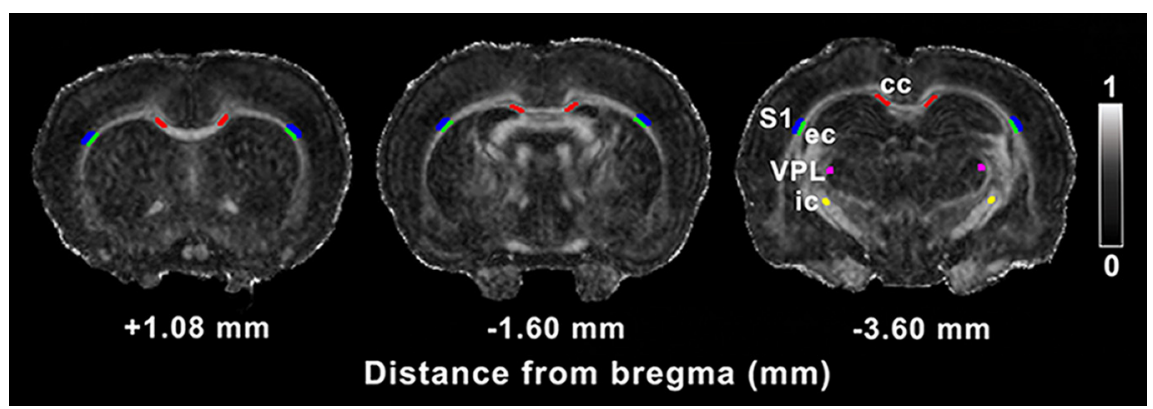

Figure 1. ROls included in the DTI analysis. ROls are outlined in a representative coronal FA map of a sham-operated animal. Gray scale indicates FA values between 0 (black) and 1 (white). cc, corpus callosum; ec, external capsule; ic, internal capsule; S1, somatosensory cortex; VPL, ventral posterolateral thalamic nucleus.

described above. The brain areas included in the ROI analysis were the ipsilateral and contralateral corpus callosum, external capsule, Layer VI of somatosensory cortex, internal capsule, and ventral posterolateral thalamic nucleus (Fig. 1). From each ROI, we extracted the FA and $A D$ on day 28 post-mTBI as the only DTI parameters with statistically significant differences at this time point. The ROIs were outlined on FA maps from each individual animal using in-house MATLAB code (AEDES, http://aedes. uef.fi/; MATLAB R2012b).

T2-weighted data were analyzed using deformationbased morphometry to assess local morphologic volume differences throughout the brain (Gaser et al., 2001). We created a study-specific T2-weighted template image (the mean of six accurately registered sham-operated and mTBI brain images). Then, the individual images were coregistered to the template using ANTs linear and nonlinear SyN registration. To determine the changes in each voxel, we computed the Jacobian determinant by the inverse displacement vector field from the SyN registration using the CreateJacobianDeterminantImage script of ANTs. In this analysis, we compared sham-operated and $\mathrm{MTBI}$ rats on days 3 and 28 using FSL randomize.

At $3 \mathrm{~d}$ post-mTBI, we observed that the in vivo T2weighted and DTI data showed blood-related susceptibility difference-artifacts related to the surgical procedure in seven sham-operated and $\mathrm{mTBI}$ animals, preventing satisfactory co-registration of some brain volumes for the T2-weighted deformation-based morphometry and DTI voxel-wise statistical analyses. From the 25 animals mentioned above $\left(n_{\text {total }}=25\right)$, we excluded six animals from the T2-weighted analysis $(n=19)$ and seven animals from the in vivo DTI analysis on day 3 post-mTBI $(n=18)$. For the same reason, on day 28 post-mTBI, we excluded three animals from the T2-weighted data analysis $(n=22)$ and two animals from the in vivo DTI analysis $(n=23)$.

\section{Tissue processing and histology}

Thirty-five days post-mTBI, we anesthetized all rats with an intraperitoneal injection of urethane $(1.25 \mathrm{~g} / \mathrm{kg}$, i.p., Sigma-Aldrich). Next, the animals were transcardially perfused with $0.9 \% \mathrm{NaCl}$ for $5 \mathrm{~min}(30 \mathrm{ml} / \mathrm{min})$, followed by $4 \%$ paraformaldehyde in $0.1 \mathrm{M} \mathrm{PB}, \mathrm{pH} 7.4$, for $25 \mathrm{~min}$ (30 $\mathrm{ml} / \mathrm{min})$. The brains were removed from the skull and postfixed in 4\% PFA for $4 \mathrm{~h}$. The brains were then placed in a cryoprotective solution ( $20 \%$ glycerol in $0.02 \mathrm{~m}$ potassium PBS, pH 7.4) for 36 h. After cryoprotection, we rapidly froze the brains in dry ice and stored them at $-70^{\circ} \mathrm{C}$ until cutting. Using a sliding microtome, we sectioned a subgroup of brains (sham-operated $=6 ; \mathrm{mTBI}=8$ ) in the coronal plane (30 $\mu \mathrm{m}, 1$-in- 5 series). We stored the first series of sections in $10 \%$ formalin, and the remaining series in cryoprotectant tissue-collecting solution (30\% ethylene glycol, $25 \%$ glycerol in $0.05 \mathrm{~m}$ sodium phosphate buffer) at $-20^{\circ} \mathrm{C}$ until histologic processing.

For the first series of sections, we performed Nissl staining (thionin) to assess the cytoarchitectonics, gliosis [increased cell density (CD)], and neurodegeneration after mTBI. In the second series of sections, we used gold chloride (Laitinen et al., 2010) to assess the myelinoarchitecture, myelin content, and axonal damage of the brain areas affected after mTBI. Briefly, sections mounted on gelatin-coated slides were incubated in $0.2 \%$ gold chloride solution $\left(\mathrm{HAuCl}_{4} \cdot 3 \mathrm{H}_{2} \mathrm{O}, \mathrm{G}-4022\right.$ MilliporeSigma) in $0.02 \mathrm{M}$ sodium phosphate buffer $(\mathrm{pH} 7.4)$ containing $0.09 \% \mathrm{NaCl}$ for $3-4 \mathrm{~h}$ at room temperature in the dark. We then washed the slides twice (4 min each) in $0.02 \mathrm{M} \mathrm{so-}$ dium phosphate buffer containing $0.09 \% \mathrm{NaCl}$ and incubated them in $2.5 \%$ sodium thiosulfate solution $(5 \mathrm{~min})$. After three washes in the buffer solution (10 min each), the slides were dehydrated $(13 \mathrm{~min})$, cleared in xylene (15 min), and cover-slipped with DePeX (Merck Millipore).

\section{ST analysis of myelin-stained sections}

For the ST analysis, high-resolution photomicrographs of myelin-stained sections were acquired at a resolution of $0.013 \mu \mathrm{m}^{2} / \mathrm{pixel}$ using a light microscope (Zeiss Axio Imager2) equipped with a digital camera (Zeiss Axiocam color 506). Each photomicrograph was analyzed using the ST-based method (Budde and Frank, 2012). To derive the anisotropy from the image, we used pixelwise ST analysis, where directional derivatives of an image were first produced by convolving the image with the directional derivative of a 2D Gaussian function (size $=11$ pixels, $\sigma=3$ pixels). Then, the ST for each image pixel was formed from the derivatives. These STs were summed into a pixelwise ST within a $128 \times 128$-pixel window, and the pixelwise tensor was eigen-decomposed. We used the eigenvalues to calculate the anisotropy index (Al). To compare the Al derived from ST-based analysis to the FA 
derived from DTI, we performed similar ROI analyses of the DTI data on myelin-stained sections, selecting the same brain areas at the locations mentioned above for the DTI data (Fig. 1). In the multiple linear regression analyses, we correlated MRI and histologic parameters obtained from white and gray matter areas individually and also using combinations of connected white and gray matter areas.

\section{CD analysis on Nissl-stained sections}

We acquired high-resolution photomicrographs of the Nissl-stained sections and analyzed all the images using an in-house MATLAB code for automated cell counting analysis. Briefly, we developed an automated cell counting technique based on preliminary foreground segmentation and a subsequent segmentation error-correction strategy. For the preliminary cell segmentation, we applied Chan-Vese active contours (Chan and Vese, 2001) as implemented in MATLAB. The Chan-Vese model was initialized with a random binary mask. The speed function was the gradient of the green channel of the RGB images; the cell membranes appeared sharper in the green channel than in the blue and red channels. We set the smooth factor equal to 0.2 , and the maximum number of iterations to 300 to perform the segmentation. The initial contours were deformed on the speed function to adapt to the cell shape, resulting in the preliminary segmentation of cells. The preliminary segmentation, however, contained undersegmented components (cells touching each other appear in the same connected component) and very small noise components. Thus, we fitted an ellipse to every preliminary component. If the major axis of an ellipse was smaller than a threshold [S=30], the component was discarded, and if the major axis was greater than a threshold $[B=140]$, the component was recognized as an undersegmented component. The preliminary segmented components with the major axis of the fitted ellipse in [S, B] were correctly segmented, requiring no further analysis. We ran a secondary segmentation on every preliminary under-segmented component using the marker-based watershed transform. We determined the markers by first Gaussian filtering the intensity image obtained from the HSV transform of the RGB image. Within the domain of an under-segmented component, we defined the regional maxima of the $\mathrm{H}$-maxima transform of the filtered image as markers. We applied a set of conditions to the extracted markers: (1) the value of a marker on the intensity image should be greater than a threshold [I=0.5]; (2) the spatial distance between two markers should be greater than a threshold $\left[D_{s}=70\right]$; and (3) the intensity distance between two markers should be greater than a threshold $\left[D_{i}=1\right]$. Determining the set of markers, we applied a watershed transform to finalize the cell segmentation. We set the thresholds to experimentally evaluate the performance of the automated cell segmentation compared with 10 images in which all cells were counted by an expert (ISMM). We used the same threshold values for all images from different brain regions and experimental conditions. We defined the CD as $\rho=\mathrm{N} / \mathrm{A}$, where $N$ is the number of cells in the ROI and A is the area of the ROI.

\section{Statistical analyses of the histology}

ROls statistical analyses of ST and CD histologic parameters were performed with GraphPad Prism (version 5.03, GraphPad Software Inc.). All values were expressed as mean $\pm \mathrm{SD}$. The unpaired $t$ test was used to assess differences between both sham-operated and $\mathrm{mTBI}$ animals. Multiple linear regression was used to assess the relationship between DTI (FA and $A D)$ and the histologic (Al and CD) parameters (regression model: DTI parameter $\sim \mathrm{Al}+\mathrm{CD}$ ). Multiple comparison correction was applied based on false discovery rate (FDR) using BenjaminiHochberg procedure (Benjamini and Hochberg, 1995). To provide more information than just significant and nonsignificant division, we computed FDR-adjusted $p$ values that we call $q$ values according to the usual terminology. The FDR-adjustment was performed using the standard procedure introduced by Yekutieli and Benjamini (1999). We applied the FDR to correct for multiple comparisons separately in the unpaired $t$ test and the multiple linear regression $p$ values. The threshold for statistical significance was set to $q<0.05$.

\section{Results}

Three days after the injury, eight of $13 \mathrm{mTBI}$ rats showed hyperintensity indicative of edema at the injury site in the somatosensory cortex at $-3.60 \mathrm{~mm}$ from bregma on T2-weighted images. Additionally, two of these eight rats also showed hypointensity in the cortex, indicating the presence of bleeding. In the subacute phase, six of 13 rats showed persistent cortical edema, but it was less pronounced compared with that at $3 \mathrm{~d}$ post-mTBI. Three of these six rats also showed bleeding.

\section{Whole-brain voxel-wise group analysis of in vivo DTI in the acute phase of $\mathrm{mTBI}$}

At day 3 post-mTBI, voxel-wise group analysis revealed a decrease in the DTI parameters in both the gray and white matter, mainly ipsilateral to the injury. Rostrally, at $-1.60 \mathrm{~mm}$ from bregma, we observed significant differences between the sham-operated and mTBI groups (Fig. 2). At this level, we observed significant decreases in FA, $A D$, and $M D$ along the external capsule up to the cingulum. The $A D$ and $M D$ were significantly different between groups in the white matter, including the corpus callosum and fimbria, and in the gray matter in the somatosensory cortex, whereas differences in RD were only observed in the somatosensory cortex (Fig. 2). At the epicenter of the lesion, at $-3.60 \mathrm{~mm}, \mathrm{FA}, \mathrm{AD}$, and MD were decreased in white matter areas: the external capsule, corpus callosum, internal capsule, and cingulum. In gray matter areas, all the parameters were decreased in the somatosensory cortex, auditory cortex, and hippocampus (Fig. 2). Also, $\mathrm{CL}$ and CS significantly differed between groups in the corpus callosum, external capsule, internal capsule, and somatosensory cortex, whereas significant differences in $\mathrm{CP}$ were only observed in the external capsule (Fig. 3). At $-4.60 \mathrm{~mm}$ from bregma, in addition to the structures mentioned at the previous level, the corpus callosum showed a significant ipsilateral decrease that extended to 


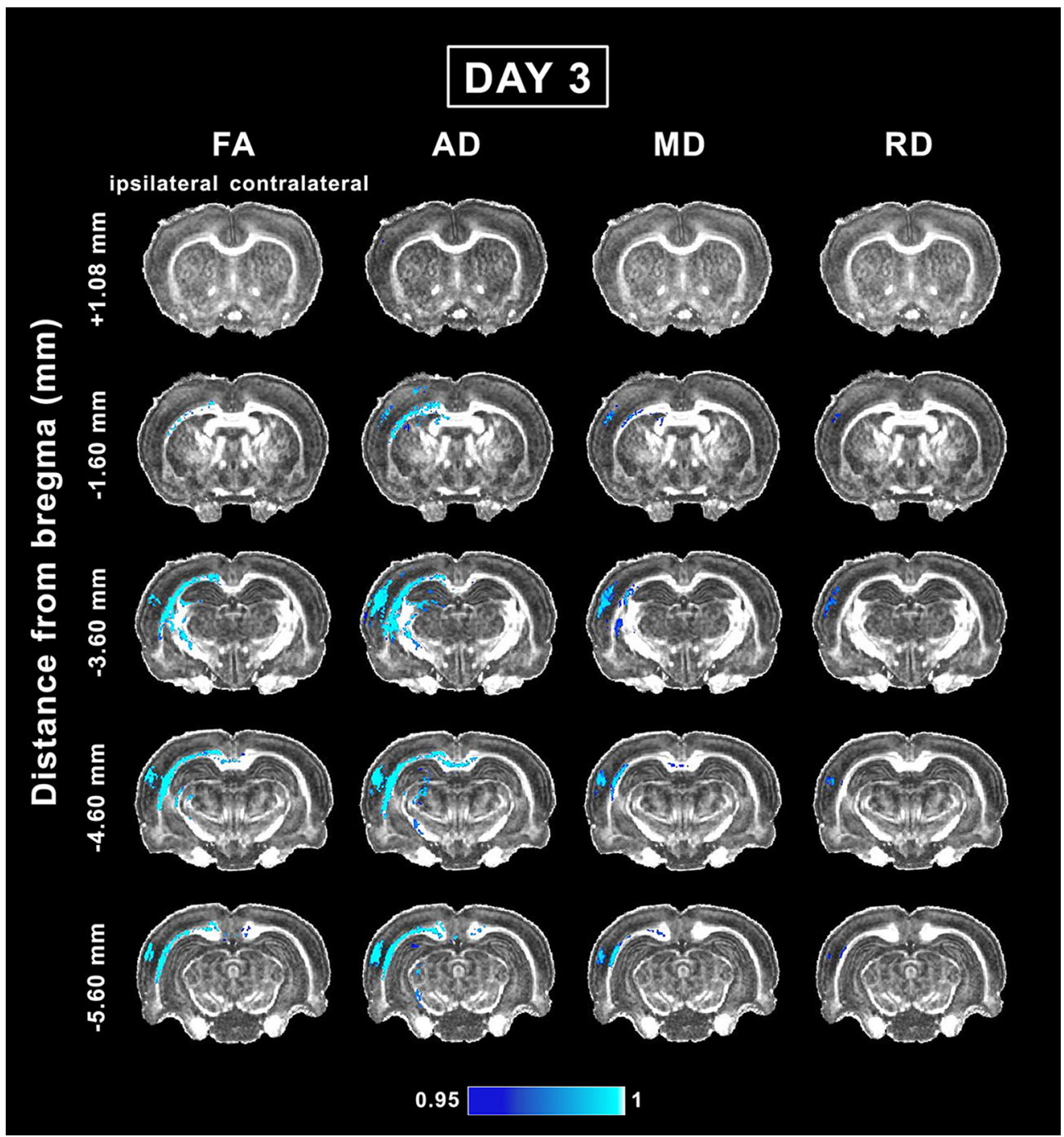

Figure 2. Whole-brain voxel-wise group analysis of $F A, A D, M D$, and RD parameters comparing sham-operated and mTBI animals at day 3. The mTBI rats showed significantly reduced FA, AD, MD, and RD parameters. The figure shows $1-p$, where $p$ is the permutation-based FWE corrected $p$ value after TFCE enhancement of the test statistic; a corrected $p<0.05$ was considered significant (blue-light blue color scale). AD, axial diffusivity; FA, fractional anisotropy; MD, mean diffusivity; RD, radial diffusivity.

the contralateral side. Furthermore, the ventrobasal complex was highlighted in the $\mathrm{FA}, \mathrm{AD}, \mathrm{CL}$, and $\mathrm{CS}$ analyses (Figs. 2, 3). More caudally at $-5.60 \mathrm{~mm}$, FA, $A D$, and $M D$ were decreased in the external capsule, corpus callosum, and auditory cortex. AD was also decreased in the hippocampus and midbrain. Similarly to the previous level, RD was only decreased in the auditory cortex (Fig. 2). Furthermore, CL and CS exhibited significant differences in the corpus callosum, external capsule, and auditory cortex, whereas significant differences in the CP were only observed in the external capsule (Fig. 3).

\section{Whole-brain voxel-wise group analysis of in vivo DTI in the subacute phase of $\mathrm{MTBI}$}

At day 28 post-mTBI, voxel-wise group analysis revealed a decrease in DTI parameters mainly in the white matter ipsilateral to the injury. Overall, we observed no progression of the abnormalities between days 3 and 28 . Rostrally in the brain, the analysis showed significant differences between groups at $-1.60 \mathrm{~mm}$ from bregma (Fig. 4). At this level, the external capsule and cingulum exhibited a decrease in $F A, A D, C L$, and $C S$ (Figs. 3, 4). At $-3.60 \mathrm{~mm}, \mathrm{FA}$ and $\mathrm{AD}$ were decreased in the corpus callosum, cingulum, and external capsule. FA was also decreased in the internal capsule and fimbria (Fig. 4). CL and CS were also significantly different between groups in the corpus callosum, external capsule, cingulum, and internal capsule (Fig. 3). Caudally at $-4.60 \mathrm{~mm}, \mathrm{FA}, \mathrm{AD}, \mathrm{CL}$, and CS were significantly decreased in the external capsule and cingulum. In addition, FA and CL were decreased in the corpus callosum ipsilaterally and contralaterally (Figs. 3, 4). At $-5.60 \mathrm{~mm}$ from bregma, FA, CL, and CS were decreased in the corpus callosum and external capsule. Additionally, AD and $\mathrm{CP}$ exhibited significant differences in the external capsule (Figs. 3, 4). 


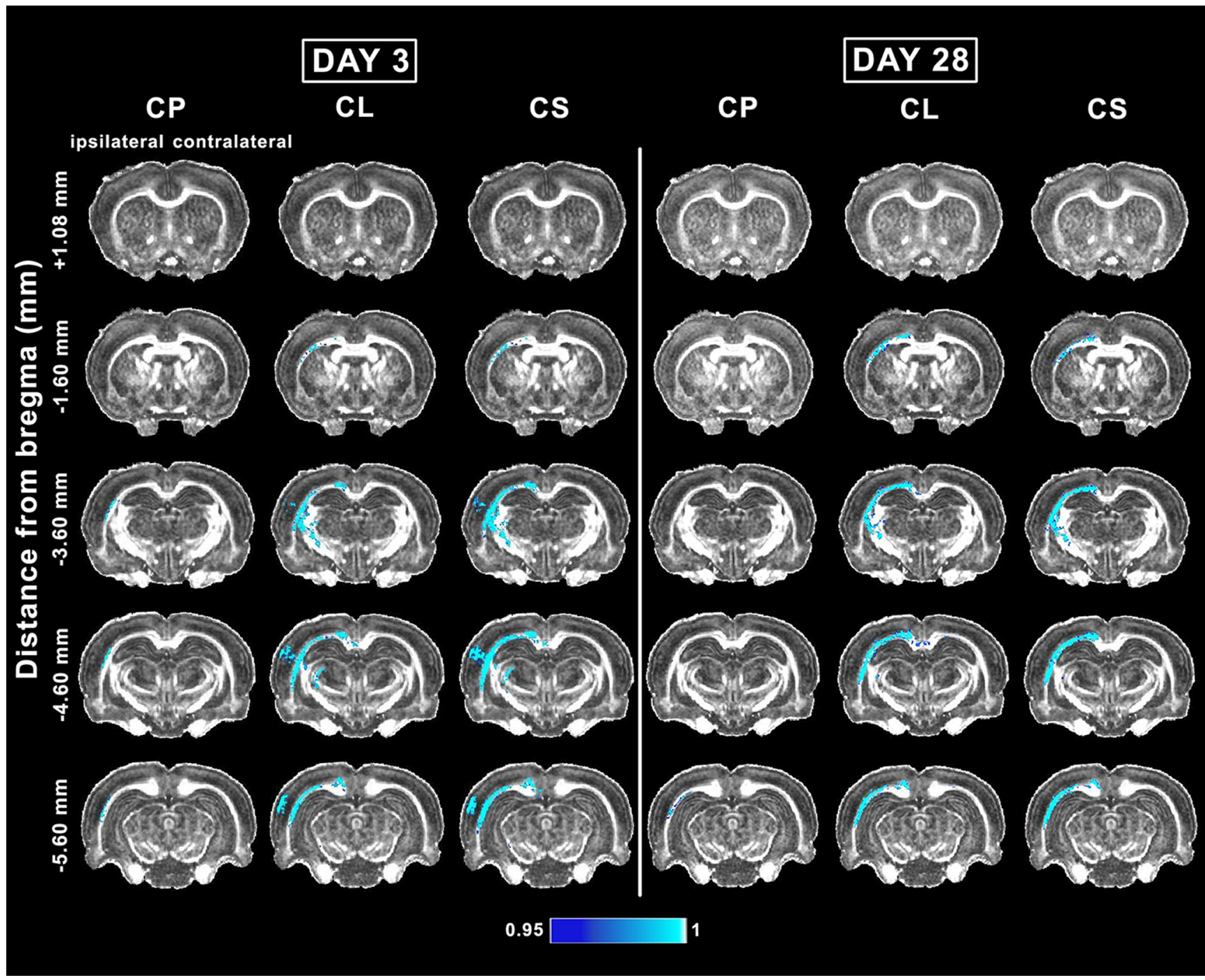

Figure 3. Whole-brain voxel-wise group analysis of Westin's derived DTI parameters comparing sham-operated and mTBI animals at 3 and $28 \mathrm{~d}$. The mTBI rats showed significantly reduced Westin's derived DTI parameters. The figure shows $1-p$, where $p$ is the permutation-based FWE corrected $p$ value after TFCE enhancement of the test statistic; a corrected $p<0.05$ was considered significant (blue-light blue color scale). CL, linear anisotropy; CP, planar anisotropy; CS, spherical anisotropy indices.

\section{T2-weighted deformation-based morphometry analysis after $\mathrm{mTBI}$}

To examine the intensity differences in T2-weighted images between sham and mTBI groups, we also performed intensity-based statistical analyses, and found no significant difference $(p=0.05$, FWE-corrected TFCE-enhanced statistics).

At day $3, \mathrm{mTBI}$ animals showed decreased volume contralaterally in the lateral ventricle in the more rostral levels (Fig. 5A). At the epicenter of the lesion, the volume was increased in the ipsilateral external capsule and Layer $\mathrm{VI}$ of somatosensory cortex, and decreased in the ventrobasal complex after mTBI. At $-4.60 \mathrm{~mm}$, mTBI animals exhibited decreased volume ipsilaterally in the dorsolateral geniculate nucleus, and increased volume in the external capsule (Fig. 5A). Even at -5.60 $\mathrm{mm}$ from bregma, we still observed an increased volume in the external capsule.
At day 28 post-mTBI (Fig. 5B), the volume changes ipsilateral to the injury were more widespread compared with day 3 (Fig. 5A). In addition, the volume changes observed at day 3 were further progressed at day 28. Rostrally, mTBI animals exhibited decreased volume in the corpus callosum, cingulum, and caudate putamen. At $-1.60 \mathrm{~mm}$ from bregma, the volume was decreased in the internal capsule, fimbria, and motor cortex (Fig. 5B). At -3.60 $\mathrm{mm}, \mathrm{mTBI}$ animals showed decreased volume in the internal capsule, dentate gyrus, somatosensory cortex, and ventrobasal complex. At $-4.60 \mathrm{~mm}$, the volume was decreased in the cingulum, corpus callosum, ventrobasal complex, and somatosensory and auditory cortex (Fig. $5 B$ ). At $-5.60 \mathrm{~mm}$ from bregma, mTBI animals exhibited decreased volume in the corpus callosum and dentate gyrus, and in the somatosensory, auditory and visual cortices. At this level, the medial geniculate nuclei showed an increased volume after mTBI. 


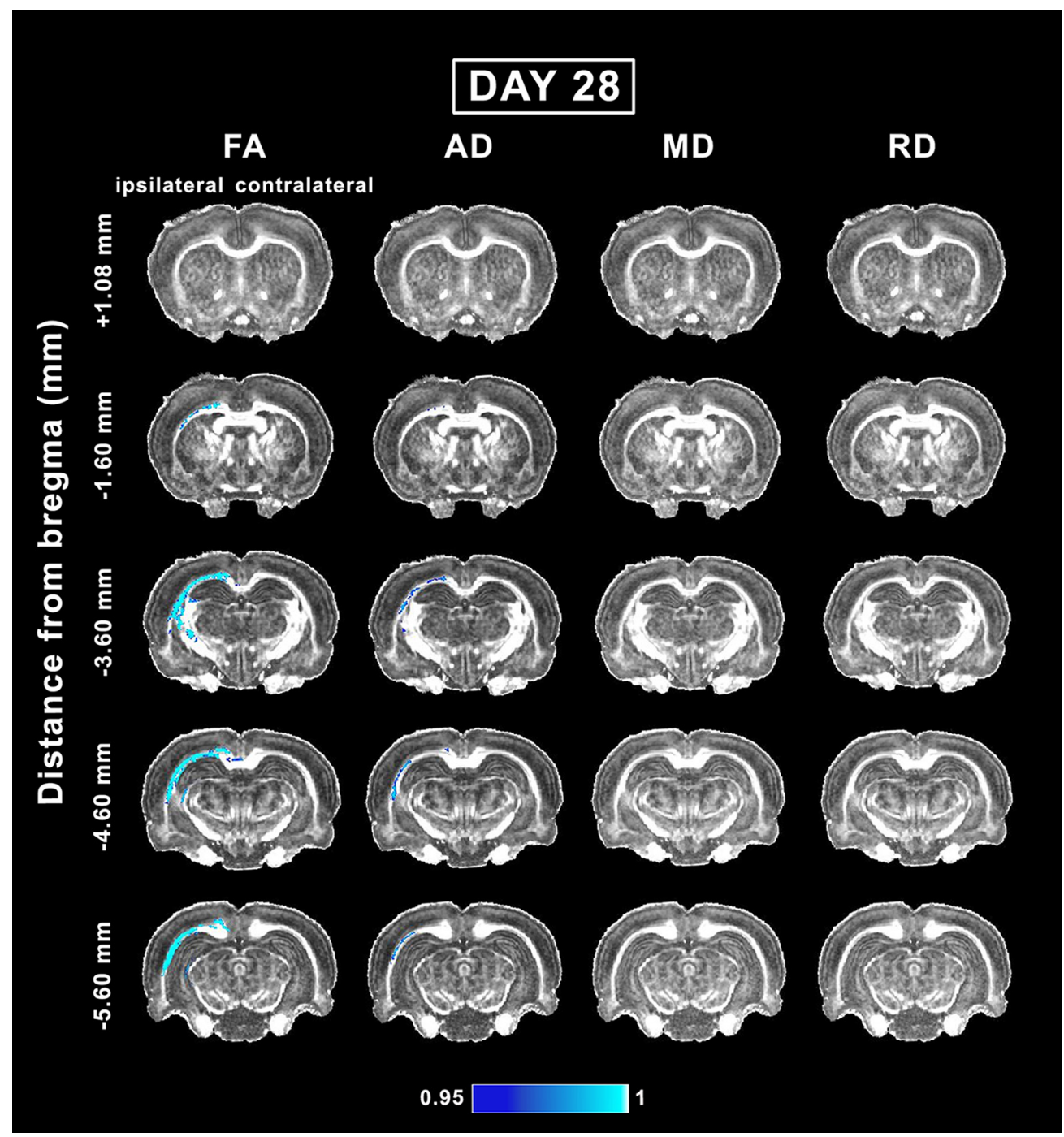

Figure 4. Whole-brain voxel-wise group analysis of $F A, A D, M D$, and RD parameters comparing sham-operated and mTBI animals at day 28. The mTBI rats showed significantly reduced FA, AD, MD, and RD parameters. The figure shows $1-p$, where $p$ is the permutation-based FWE corrected $p$ value after TFCE enhancement of the test statistic; a corrected $p<0.05$ was considered significant (blue-light blue color scale). AD, axial diffusivity; FA, fractional anisotropy; MD, mean diffusivity; RD, radial diffusivity.

\section{Qualitative and quantitative histologic assessment of myelin and Nissl-stained sections in the subacute phase of $\mathrm{mTBI}$}

For histologic assessment, we focused our analysis on areas highlighted in the group analyses, including the corpus callosum, external capsule, layer VI of somatosensory cortex, internal capsule, and ventral posterolateral thalamic nucleus both ipsilaterally and contralaterally. We analyzed these areas at two levels rostral to the lesion $(+1.08$ and $-1.60 \mathrm{~mm}$ from bregma), and at the epicenter of the lesion $(-3.60 \mathrm{~mm}$ from bregma) where the group analyses showed or did not show statistically significant differences.

The most devastating consequence after TBI is diffuse axonal injury caused by rapid acceleration/deceleration movements of the head (Marion et al., 2018). As a result, the axonal cytoarchitecture changes, progressing from a disruption in axonal transport to axonal swelling, secondary disconnection, and, finally, demyelination or Wallerian degeneration (Johnson et al., 2013). The alterations in myelinated axons at $35 \mathrm{~d}$ after injury may correspond with injured or degenerating axons, or demyelination.

Together with a qualitative examination of the histologic preparations, we performed quantitative assessments in both ipsilateral and contralateral hemispheres to compare sham-operated $(n=6)$ and mTBI animals $(n=8)$ by using ST analysis and automated cell counting on myelinstained and Nissl-stained sections at day 35 post-mTBI. The results of the analysis are summarized in Table 1. We did not find any qualitative or quantitative differences when comparing histologic preparations from the contralateral side of sham-operated and $\mathrm{mTBI}$ animals (Table 1). Here, we focus our description on the comparison of the ipsilateral hemisphere between sham-operated and mTBI animals. 


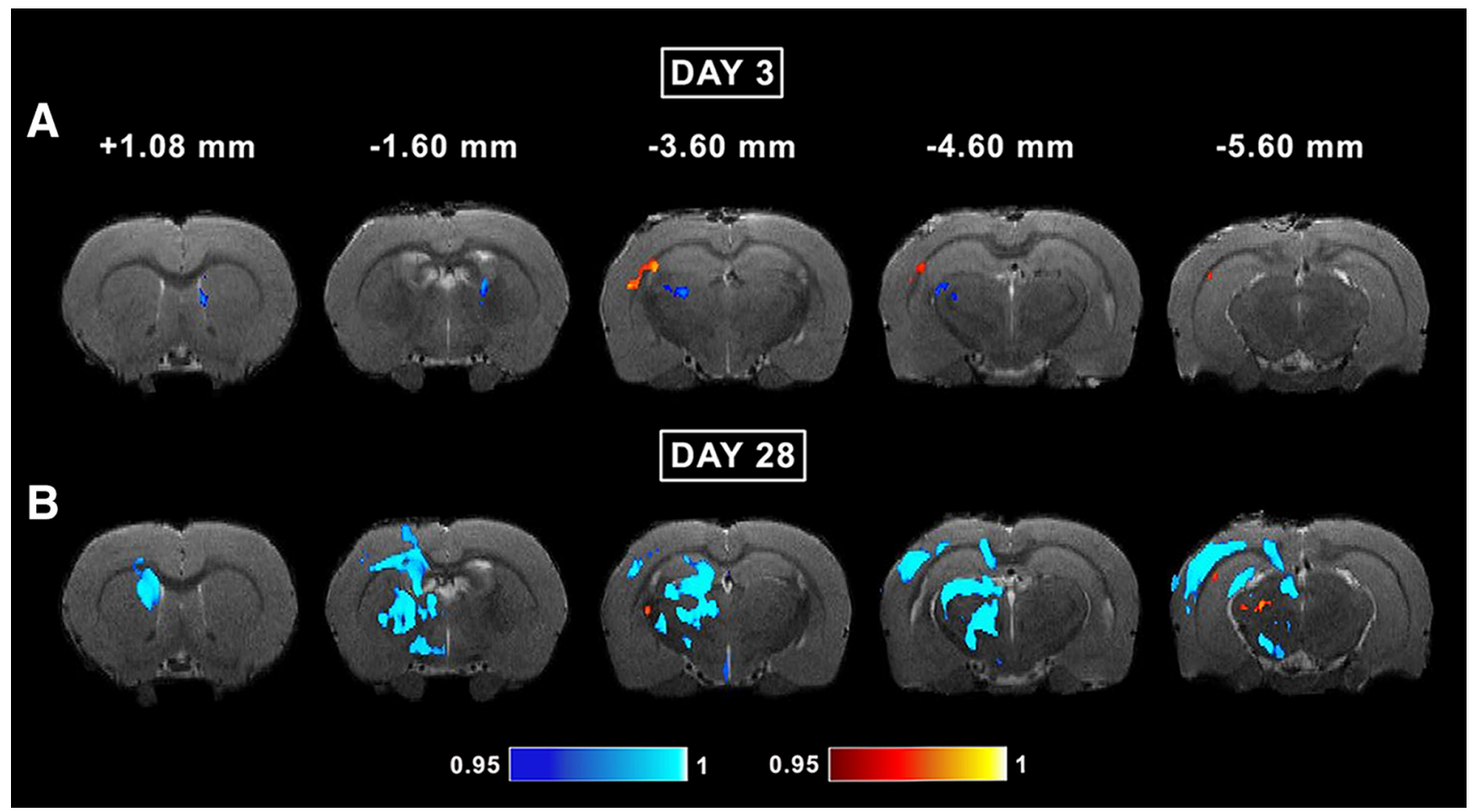

Figure 5. Whole-brain group deformation-based morphometry analysis of T2-weighted images comparing sham-operated and $\mathrm{mTBI}$ animals at day $3(\boldsymbol{A})$ and $28(\boldsymbol{B})$. Brain volume differences between sham-operated and mild TBI animals observed in acute and subacute phases post-mTBI. The mTBI rats showed both volume enlargement (red-yellow color scale), and volume reduction (blue-light blue color scale) compared with the sham-operated rats. The figure shows $1-p$, where $p$ is the permutation-based FWE corrected $p$ value after TFCE enhancement of the test statistic; a corrected $p<0.05$ was considered significant.

Rostrally, at $+1.08 \mathrm{~mm}$, we observed alterations along myelinated axons in the ipsilateral external capsule and somatosensory cortex after mTBI (Fig. 6Cii,Dii), with no changes in cellularity (Fig. 6Civ,Div). Ipsilaterally in the corpus callosum, however, we observed no changes in the myelin or cellularity between mTBI and sham-operated animals (Fig. 6Bi-iv). We did not find significant differences between sham-operated and mTBI animals in any of these areas (Table 1).

At $-1.60 \mathrm{~mm}$ from bregma, we observed more pronounced tissue alterations than at $+1.08 \mathrm{~mm}$. Here, mTBI animals showed mild axonal damage and gliosis in the corpus callosum (Fig. 7Bii-iv). We observed axonal damage and gliosis after mTBI in the external capsule and the somatosensory cortex (Fig. 7C,Dii-iv). Although some values showed a trend, especially the external capsule with lower $\mathrm{Al}$ and higher $\mathrm{CD}$ values comparing animal groups, we did not find significant differences between sham-operated and mTBI animals in any of these areas (Table 1).

The analyzed areas showed more extensive alterations at the epicenter of the lesion than rostrally. In the corpus callosum, we observed widespread axonal damage (Fig. 8 Bii) and increased cellularity (Fig. 8Biv) after mTBl; however, no significant differences were found in Al or CD when comparing sham-operated and $\mathrm{mTBI}$ animals (Table 1). In addition to the pronounced and widespread axonal damage, we observed a loss of myelinated axons and increased cellularity in the ipsilateral external capsule and somatosensory cortex in mTBI animals (Fig. 8C,Dii- iv) as compared with sham-operated animals (Fig. 8C,Diiii). In the external capsule, we found a significant decrease in $\mathrm{Al}\left(t=4.97, q=1.76 \times 10^{-2}\right)$ which corroborated the damage and loss of myelinated axons, and a significant increase in CD $\left(t=4.71, q=1.76 \times 10^{-2}\right)$ along with gliosis when comparing $\mathrm{mTBI}$ and sham-operated animals (Table 1). In the somatosensory cortex, we only found a significant increase in CD $(t=4.01, q=3.41 \times$ $10^{-2}$ ) corresponding with the presence of gliosis after mTBI (Table 1). Furthermore, in the ipsilateral internal capsule and ventral posterolateral thalamic nucleus, we observed axonal damage (Fig. 8Eii,Fii) and increased cellularity (Fig. 8Eiv,Fiv) after mTBI. We found a significant increase in $C D$ in the internal capsule, indicative of gliosis in $\mathrm{mTBI}$ animals when comparing to sham-operated $\left(t=4.23, q=3.41 \times 10^{-2}\right.$; Table 1$)$.

\section{Multiple linear regression of DTI and histologic parameters}

We assessed the relationship between the DTI and histologic parameters from the ST and CD analyses in white and gray matter using a multiple linear regression test. Our regression model evaluated the contribution of two factors, $\mathrm{Al}$ and $\mathrm{CD}$, to $\mathrm{FA}$ or $\mathrm{AD}$ (DTI parameter $\sim \mathrm{Al}+$ $\mathrm{CD})$. Table 2 summarizes the outcomes of the multiple linear regression test, which includes the statistics of the model ( $R^{2}$ adjusted and $F$ statistic), the $t$ statistics for Al and $C D[t(A l)$ and $t(C D)]$ and the corresponding $q$ values. 
Table 1: Quantitative histologic analysis at $+1.08,-1.60$, and $-3.60 \mathrm{~mm}$ from bregma

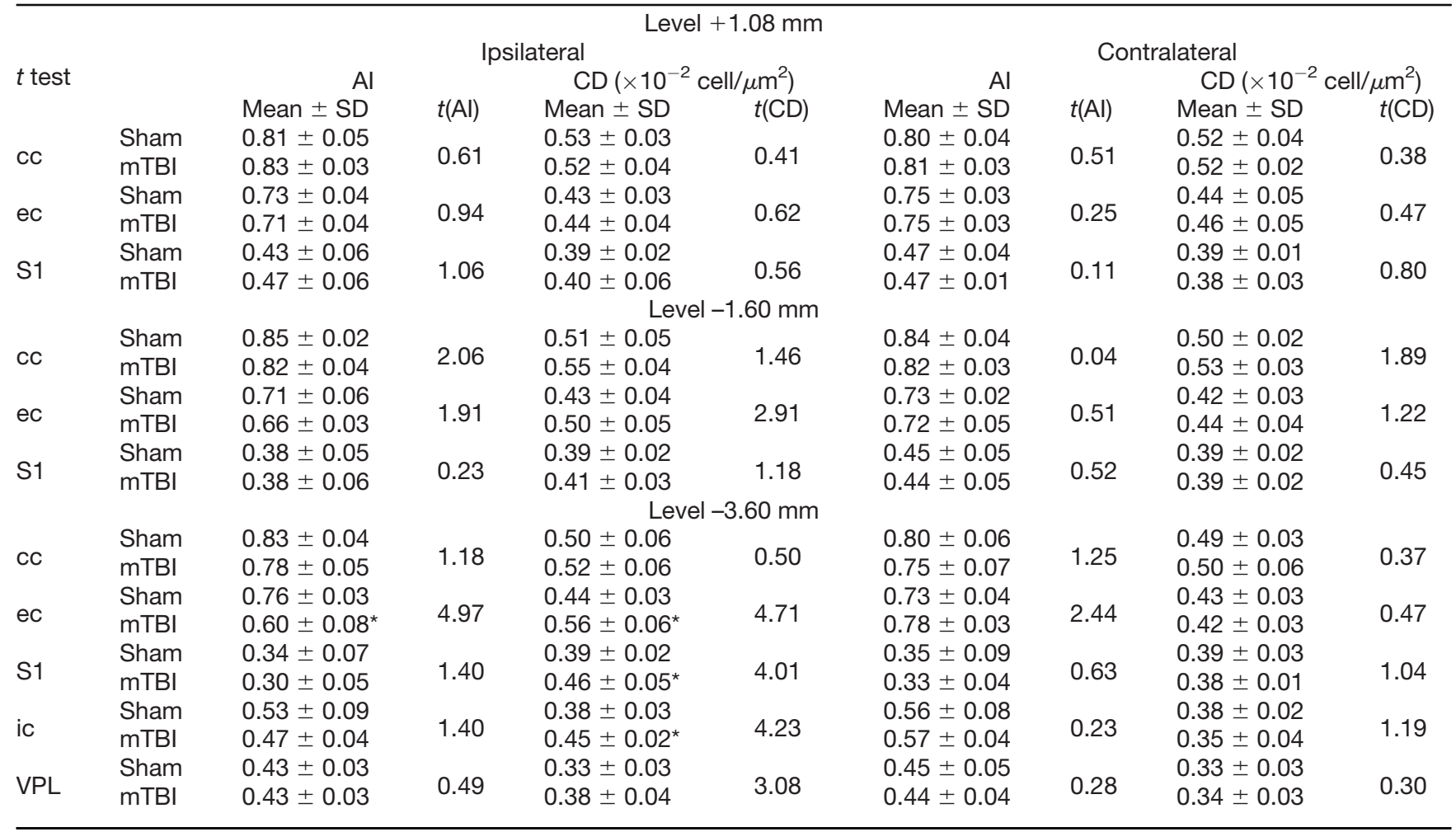

Statistically significant FDR-corrected $q$ values are shown $\left({ }^{*} q<0.05\right.$ corresponding to uncorrected ${ }^{*} p<3.10 \times 10^{-3}$; unpaired $t$ test) for the anisotropy index (Al) and CD $\left(\times 10^{-2}\right.$ cell $\left./ \mu \mathrm{m}^{2}\right)$. Al, anisotropy index; cc, corpus callosum; CD, cell density; ec, external capsule; ic, internal capsule; S1, somatosensory cortex; VPL, ventral posterolateral thalamic nucleus.

Figure 9 shows the most representative multiple linear regression results between $\mathrm{FA}$ or $\mathrm{AD}$ and $\chi=\beta_{\mathrm{Al}} \times \mathrm{Al}+$ $\beta_{\mathrm{CD}} \times \mathrm{CD}$, where $\beta$ is the weighting value.

Rostrally, at $+1.08 \mathrm{~mm}$, we found a correlation between FA and $\chi\left(R^{2}=0.68, q=2.30 \times 10^{-12}\right.$; Fig. $\left.9 A\right)$ and $A D$ and $\chi\left(R^{2}=0.66, q=3.68 \times 10^{-12}\right.$; Fig. 9B) when combining the corpus callosum and external capsule (Table 2). In these two brain areas, FA $\left(t=5.10, q=2.24 \times 10^{-5}\right)$ and AD $\left(t=6.34, q=4.55 \times 10^{-7}\right)$ were positively associated with Al. We also found positive association between FA $\left(t=6.28, q=5.09 \times 10^{-7}\right)$ and $\mathrm{AD}(t=4.53, q=1.36 \times$ $10^{-4}$ ), and CD (Table 2). Despite the positive association between DTI and histologic parameters with AI and CD, we observed low influence of the injury in the correlations (Fig. 9A,B).

At $-1.60 \mathrm{~mm}$ from bregma, FA correlated with $\chi$ in the corpus callosum $\left(R^{2}=0.51, q=2.13 \times 10^{-4}\right)$, while AD correlated with $\chi$ in the external capsule $\left(R^{2}=0.25, q=\right.$ $2.82 \times 10^{-2}$; Table 2). When combining the corpus callosum and external capsule, FA correlated with $\chi\left(R^{2}=0.33\right.$, $q=4.10 \times 10^{-5}$; Fig. $\left.9 C\right)$ and $\mathrm{AD}$ with $\chi\left(R^{2}=0.55\right.$, $q=5.39 \times 10^{-9}$; Fig. $9 D ;$ Table 2). Also, we found that both FA $\left(t=5.39, q=8.90 \times 10^{-6}\right)$ and $\mathrm{AD}(t=7.54, q=$ $\left.9.93 \times 10^{-9}\right)$ were positively associated with Al (Table 2). At this level, we observed a moderate influence of the injury in the relationship between DTI and histologic parameters, mainly in the external capsule (Fig. 9C,D).

At the epicenter of the lesion $(-3.60 \mathrm{~mm})$, we found a correlation between DTI and $\chi$ parameters in both white and gray matter areas (Table 2). In the external capsule, both FA $\left(R^{2}=0.72, q=4.55 \times 10^{-7}\right)$ and $\mathrm{AD}\left(R^{2}=0.69\right.$, $\left.q=1.11 \times 10^{-6}\right)$ correlated with $\chi$ (Table 2$)$. We found that both FA $\left(t=3.00, q=1.80 \times 10^{-2}\right)$ and $\mathrm{AD}(t=4.54$, $\left.q=4.35 \times 10^{-4}\right)$ in the external capsule were positively associated with Al (Table 2). Similarly, in the internal capsule, we also found a correlation between FA and $\chi\left(R^{2}=\right.$ $\left.0.23, q=4.13 \times 10^{-2}\right)$, and $\mathrm{AD}$ and $\chi\left(R^{2}=0.33, q=\right.$ $7.91 \times 10^{-3}$; Table 2$)$. We found that only $\operatorname{AD}(t=3.72$, $q=3.51 \times 10^{-3}$ ) was positively associated with Al (Table $2)$. When combining the corpus callosum and external capsule, FA $\left(R^{2}=0.53, q=1.04 \times 10^{-8}\right)$ and $\mathrm{AD}\left(R^{2}=\right.$ $\left.0.48, q=1.25 \times 10^{-7}\right)$ correlated with $\chi$ (Table 2). Also, we showed that both FA (Al: $t=5.02, q=2.79 \times 10^{-5}$; CD: $t=$ -4.63, $\left.q=9.88 \times 10^{-5}\right)$ and $\mathrm{AD}(\mathrm{Al}: t=5.45, q=7.50 \times$ $10^{-6}$; CD: $t=-3.17, q=8.09 \times 10^{-3}$ ) were positively associated with both histologic parameters (Table 2). When combining the three white matter areas, we found a correlation between $\mathrm{FA}\left(R^{2}=0.51, q=3.68 \times 10^{-12}\right.$; Fig. $\left.9 E\right)$ and $\mathrm{AD}\left(R^{2}=0.33, q=3.15 \times 10^{-7}\right.$; Fig. $\left.9 F\right)$ with $\chi$ (Table 2). Here, FA $\left(t=-8.52, q=1.72 \times 10^{-11}\right)$ was negatively associated with CD (Table 2). Moreover, AD was positively associated with $\mathrm{Al}\left(t=5.44, q=3.78 \times 10^{-6}\right)$ and negatively associated with $\mathrm{CD}\left(t=-5.23, q=7.49 \times 10^{-6}\right.$; Table 2). At this level, we found high influence of the injury in the correlation between DTI and histologic parameters as shown by the ipsilateral values of the three brain areas (Fig. 9E,F). In gray matter, we found that FA $\left(R^{2}=0.39\right.$, $q=4.83 \times 10^{-6}$; Fig. $\left.9 G\right)$ and $\operatorname{AD}\left(R^{2}=0.14, q=\right.$ 
A

\section{$+1.08 \mathrm{~mm}$ from bregma}
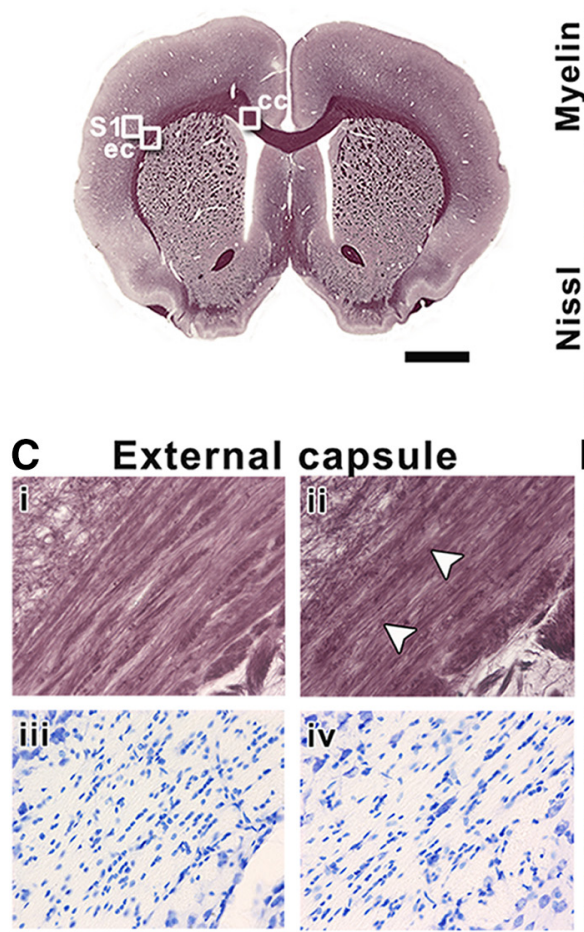
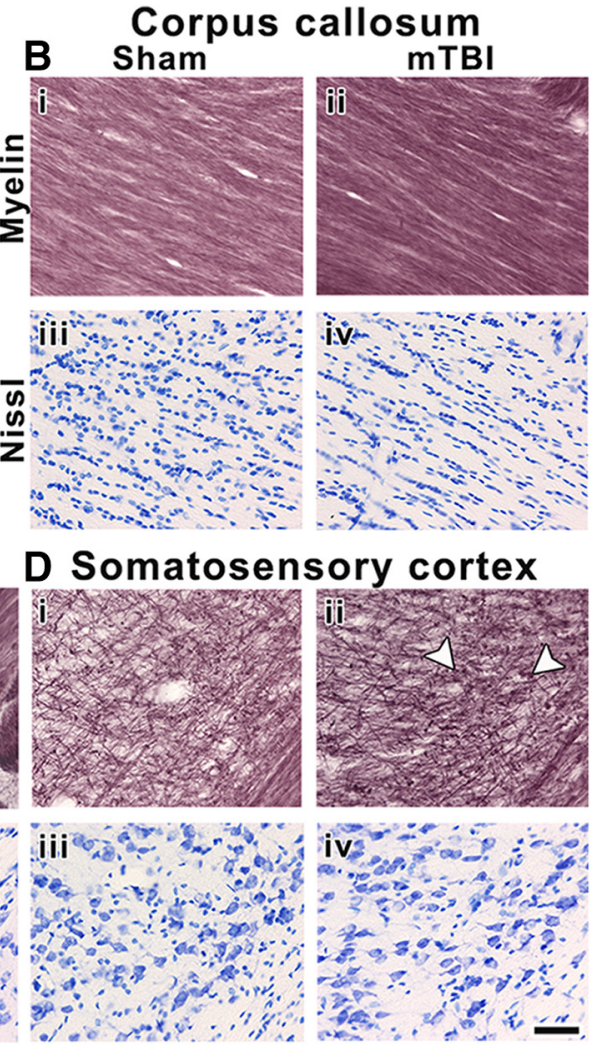

Figure 6. Representative whole-brain myelin-stained section of a sham-operated animal at $+1.08 \mathrm{~mm}$ from bregma $(\boldsymbol{A})$. White squares in $\boldsymbol{A}$ indicate the location of high-magnification photomicrographs of myelin-stained and Nissl-stained sections of a shamoperated (i and iii) and mTBI animal (ii and $\boldsymbol{i v}$ ) in the corpus callosum (B), external capsule (C), and somatosensory cortex (D). The same animals are shown in both stainings. White arrowheads indicate axonal damage. cc, corpus callosum; ec, external capsule; S1, somatosensory cortex. Scale bars: $2 \mathrm{~mm}(\boldsymbol{A})$ and $50 \mu \mathrm{m}(\boldsymbol{B}-\boldsymbol{D})$.

$1.99 \times 10^{-2}$; Fig. $9 H$ ) correlated with $\chi$ when combining the somatosensory cortex and ventral posterolateral thalamic nucleus. Both FA $\left(t=-5.12, q=2.21 \times 10^{-5}\right)$ and $\mathrm{AD}\left(t=-2.95, q=1.47 \times 10^{-2}\right)$ were negatively associated with Al (Table 2). Here, despite we found associations between DTI and histologic parameters, the influence due to the injury in these correlations was low (Fig. 9G,H).

\section{Discussion}

In the present study, we investigated the potential of in vivo DTI to detect progressive widespread microstructural tissue alterations in white and gray matter areas throughout the brain after mTBI in rats. We performed quantitative histologic characterization of the in vivo DTI findings to unveil the underlying tissue processes associated with mTBI using ST and automated cell counting analyses in the subacute phase of $\mathrm{mTBI}$. The key findings of this study were: (1) microstructural tissue alterations detected by voxel-wise analysis of DTI maps in white and gray matter areas progressed from day 3 to day 28; (2) volume reductions occur in white and gray matter areas in the subacute phase; (3) decreases in FA and AD are associated with axonal damage and gliosis in the subacute phase of $\mathrm{mTBl}$; and (4) although we found a strong correlation between DTI and histologic parameters far from the lesion site, DTI was unable to detect mild axonal damage at this level. The combination of MRI and histologic analyses increases our knowledge of the potential of in vivo DTI to detect microstructural tissue changes in the brain after mTBI. This study may provide a new window for detecting progressive mild microstructural tissue damage using in vivo DTI.

\section{Microstructural alterations in the brain after mTBI}

Three days after mTBI, our voxel-wise group analysis showed a decrease in $F A, M D, A D, C L$, and $C S$ in major white matter areas, such as the corpus callosum, external capsule, and internal capsule in the ipsilateral hemisphere. Previous studies using in vivo DTI in a rat model of $\mathrm{mTBI}$ also reported changes in $\mathrm{FA}, \mathrm{AD}, \mathrm{MD}$, and $\mathrm{RD}$ in white matter tracts in the acute phase (Long et al., 2015; Li et al., 2016; Wright et al., 2016; Herrera et al., 2017; Tu et al., 2017). Several studies report decreased FA in the corpus callosum at days 1 and 2 post-mTBI in the mild controlled cortical impact model (Long et al., 2015; Li et al., 2016; Tu et al., 2017) and increased RD (Long et al., 2015; Tu et al., 2017). Tu and collaborators also reported decreased $A D$ and increased $M D$ in the corpus callosum at day 1 post-mTBI (Tu et al., 2017). Further, using a mild impact acceleration model, Herrera and collaborators reported increased FA and MD in the fimbria and internal capsule and increased RD in the fimbria at day 3 post- 

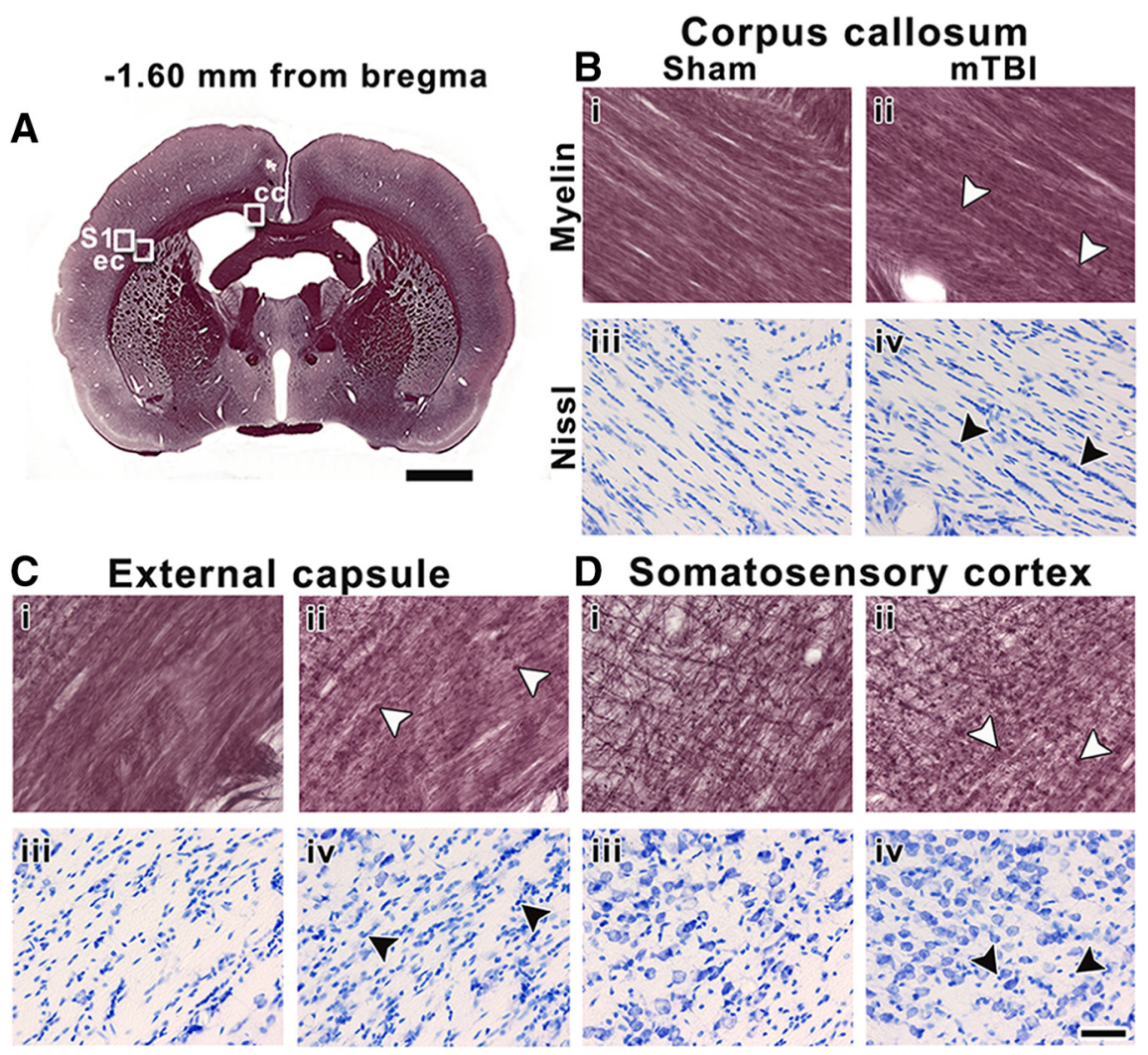

Figure 7. Representative whole-brain myelin-stained section of a sham-operated animal at $-1.60 \mathrm{~mm}$ from bregma $(\boldsymbol{A})$. White squares in panel $\boldsymbol{A}$ indicate the location of high-magnification photomicrographs of myelin-stained sections and Nissl-stained sections of a sham-operated (i and iii) and mTBI animal (ii and $\boldsymbol{i v}$ ) in the corpus callosum (B), external capsule (C), and somatosensory cortex $(\boldsymbol{D})$. The same animals are shown in both stainings. White arrowheads indicate axonal damage, and black arrowheads indicate gliosis shown by increased cellularity in Nissl-staining sections. cc, corpus callosum; ec, external capsule; S1, somatosensory cortex. Scale bars: $2 \mathrm{~mm}(\boldsymbol{A})$ and $50 \mu \mathrm{m}(\boldsymbol{B}-\boldsymbol{D})$.

mTBI (Herrera et al., 2017). Their study also showed decreased $A D$ in the genu of the corpus callosum and fimbria, and decreased RD in the splenium of the corpus callosum. Using the mild LFP injury model, another study found decreased FA in the ipsilateral corpus callosum at 3 and $5 \mathrm{~d}$ post-mTBI, and increased RD at day 5 post-mTBI (Wright et al., 2016).

Consistent with our findings, a decrease in anisotropy and diffusivities in white matter were also observed in humans in the acute phase of mTBI (Arfanakis et al., 2002; Inglese et al., 2005). These studies reported decreased FA in the corpus callosum and internal capsule in the acute phase in $\mathrm{mTBI}$ patients. Inglese and collaborators also demonstrated increased MD in the internal capsule after mTBI (Inglese et al., 2005). In summary, findings from previous studies as well as the present study reveal that major white matter areas are extensively damaged in the acute phase of mTBI (Arfanakis et al., 2002; Inglese et al., 2005; Long et al., 2015; Li et al., 2016; Wright et al., 2016; Herrera et al., 2017; Tu et al., 2017). In particular, our study demonstrated consistent decreases in both anisotropy and diffusivities in the corpus callosum, external capsule, and internal capsule.

Our findings in gray matter areas revealed decreased FA, $A D, M D$, and RD mainly in the ipsilateral somatosensory and auditory cortices in the acute phase of mTBI. This is consistent with the work of Wright and collaborators (Wright et al., 2016) who also demonstrated decreased $A D$ at 3 and $5 \mathrm{~d}$ post-mTBI in the ipsilateral cortex and increased trace at day 3 post-mTBI. While previous experimental and human studies revealed mainly white matter damage in the acute phase of $\mathrm{mTBI}$, our findings revealed damage in both white and gray matter areas at an acute time point.

Twenty-eight days after mTBI, voxel-wise group analysis revealed microstructural alterations mainly in major white matter areas detected by in vivo DTI. We detected both decreased FA and $A D$ ipsilaterally in the corpus callosum and external capsule and decreased FA in the internal capsule in the subacute phase post-mTBI. In these white matter areas, the decreases in CL and CS corroborated the decrease in FA at day 28 post-mTBI. Until now, only one study using a mild blast injury model in rats reported changes in the DTI metrics at day 30 post-mTBI using in vivo DTI (Budde et al., 2013). Laitinen et al. (2015), using a severe form of the LFP injury model, also demonstrated microstructural tissue changes in white matter areas, which included the genu of the corpus callosum, the angular bundle, and the internal capsule several months after injury. Human studies also showed progressive changes in the DTI metrics in white matter areas in the subacute phase in mild TBI patients (Rutgers et al., 2008; Messé et al., 2011). Messé et al. (2011) 

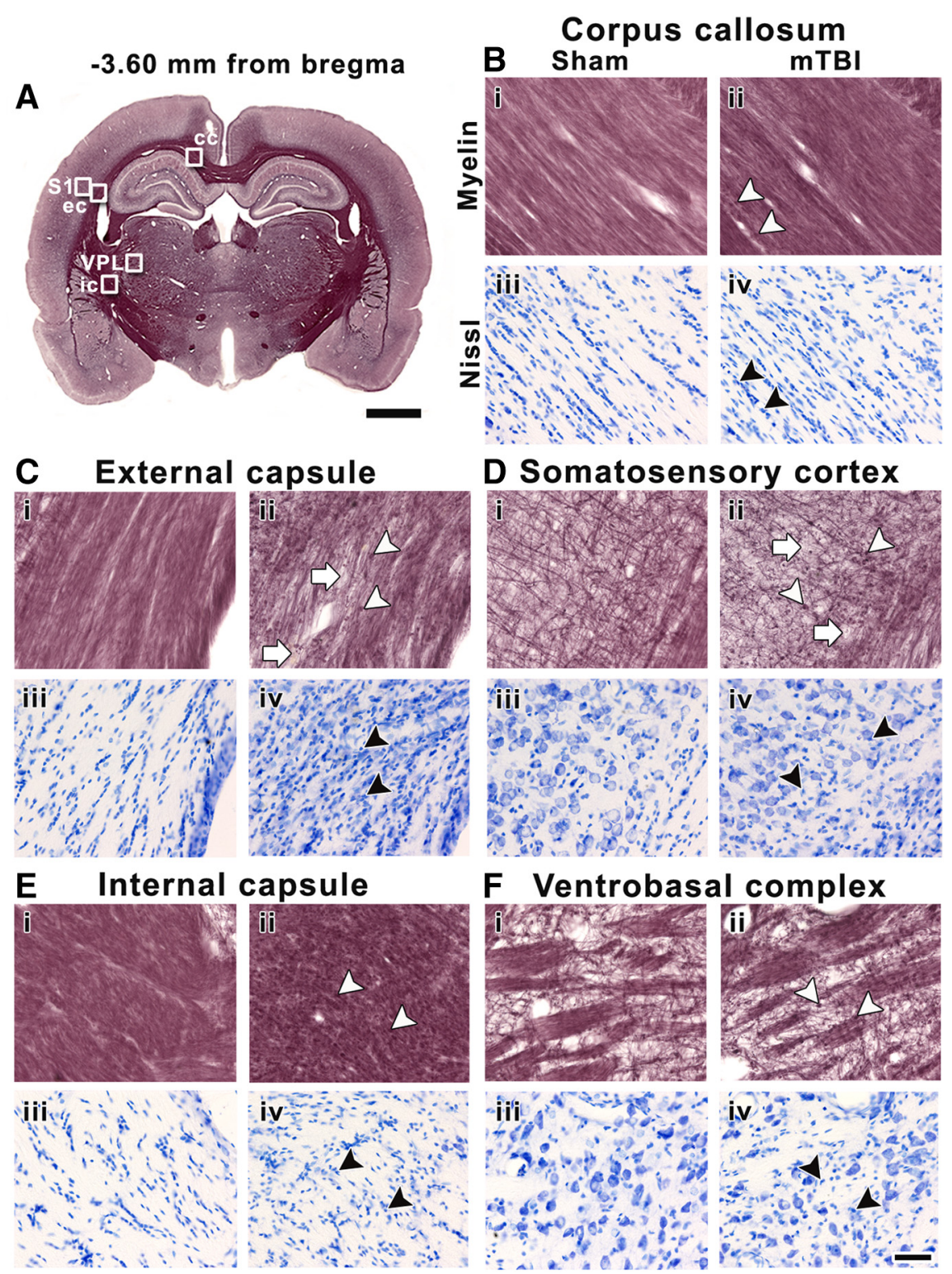

Figure 8. Representative whole-brain myelin-stained section of a sham-operated animal at $-3.60 \mathrm{~mm}$ from bregma $(\boldsymbol{A})$. White squares in panel $\boldsymbol{A}$ indicate the location of high-magnification photomicrographs of myelin-stained sections and Nissl-stained sections of a sham-operated ( $\boldsymbol{i}$ and $\boldsymbol{i i i )}$ ) and $\mathrm{mTBI}$ animal (ii and $\boldsymbol{i} \boldsymbol{V}$ ) in the corpus callosum $(\boldsymbol{B})$, external capsule $(\boldsymbol{C})$, and somatosensory cortex $(\boldsymbol{D})$, internal capsule $(\boldsymbol{E})$, and ventrobasal complex $(\boldsymbol{F})$. The same animals are shown in both stainings. White arrowheads indicate axonal damage, and white arrows point to loss of myelinated axons. Black arrowheads indicate gliosis shown by increased cellularity in Nissl-staining sections. cc, corpus callosum; ec, external capsule; ic, internal capsule; S1, somatosensory cortex; VPL, ventral posterolateral thalamic nucleus. Scale bars: $2 \mathrm{~mm}(\boldsymbol{A})$ and $50 \mu \mathrm{m}(\boldsymbol{B}-\boldsymbol{F})$.

reported higher MD values in the corpus callosum in the subacute phase of mTBI between days 7 and 28 postmTBI. In contrast, Rutgers et al. (2008) showed reduced FA predominantly in the corpus callosum and cingulum during the subacute and chronic phases of mTBI. In accordance with previous experimental and human studies, our results emphasize the persistent damage in white matter areas in the subacute phase of mTBI.

\section{Volumetric changes in the brain after mTBI}

After TBI, neuronal damage induces anatomic as well as cellular morphology changes, leading to neurodegeneration
(Harris et al., 2019). Neurodegeneration is associated with progressive volume loss after TBI detected by volumetric MRI measurements (Bigler, 2013; Cole et al., 2018). In our study, we assessed volume changes using T2-weighted deformation-based morphometry analysis. Our results revealed a volume reduction contralaterally in the lateral ventricle and ipsilaterally in the ventrobasal complex and increased volume in the external capsule and somatosensory cortex in the acute phase of mTBI. Previous studies revealed that a volume increase might be associated with edema early after mTBI (Mckee and Daneshvar, 2015; Stokum et al., 2016). In the subacute phase of $\mathrm{mTBI}$, our results mainly showed a progressive volume reduction in the gray matter 
Table 2: Multiple linear regression between quantitative DTI and histologic analysis at $+1.08,-1.60$, and $-3.60 \mathrm{~mm}$ from bregma

\begin{tabular}{|c|c|c|c|c|c|c|c|c|}
\hline & & & & $+1.08 \mathrm{mr}$ & & & & \\
\hline & & & & & & $A D$ & $\left.\mathrm{~mm}^{2} / \mathrm{s}\right)$ & \\
\hline & $R^{2}$ adj & $F$ stat & $t(\mathrm{Al})$ & $t(C D)$ & $R^{2}$ adj & F stat & $t(\mathrm{Al})$ & $t(\mathrm{CD})$ \\
\hline $\mathrm{cc}$ & 0.02 & 1.24 & -1.42 & 0.14 & 0 & 0.46 & -0.20 & -0.94 \\
\hline ec & 0.11 & 2.64 & 1.38 & 1.88 & 0.15 & 3.45 & $2.58^{\star}$ & 0.58 \\
\hline S1 & 0.01 & 1.09 & -0.11 & 1.47 & 0 & 0.15 & -0.16 & -0.48 \\
\hline $\mathrm{cc}+\mathrm{ec}$ & $0.68^{\star \star \star}$ & 60.0 & $5.10^{\star \star \star}$ & $6.28^{\star \star \star}$ & $0.66^{\star \star \star}$ & 55.1 & $6.34^{\star \star \star}$ & $4.53^{\star \star \star}$ \\
\hline & & & & $-1.60 \mathrm{mn}$ & & & & \\
\hline cc & $0.51^{\star \star *}$ & 14.8 & 1.49 & $-4.97^{\star \star \star}$ & 0.09 & 2.25 & -0.42 & -2.12 \\
\hline ec & 0.08 & 2.14 & 0.14 & -1.94 & $0.25^{\star}$ & 5.52 & 2.08 & -1.87 \\
\hline S1 & 0 & 0.40 & 0.66 & -0.55 & 0.05 & 1.65 & -0.98 & -1.60 \\
\hline$c c+e c$ & $0.33^{\star \star \star}$ & 14.5 & $5.39^{\star \star *}$ & -2.40 & $0.55^{\star \star \star}$ & 34.3 & $7.54^{\star \star \star}$ & -0.28 \\
\hline & & & & $-3.60 \mathrm{~mm}$ & & & & \\
\hline $\mathrm{cc}$ & 0.20 & 4.29 & $2.91^{*}$ & -0.78 & 0 & 0.87 & 1.17 & -0.79 \\
\hline ec & $0.72^{\star \star \star}$ & 35.4 & $3.00^{*}$ & -2.37 & $0.69^{\star \star \star}$ & 31.4 & $4.54^{\star \star \star}$ & -0.32 \\
\hline S1 & 0.08 & 2.19 & -0.35 & -2.06 & 0.03 & 1.47 & -0.36 & 0.10 \\
\hline ic & $0.23^{*}$ & 4.95 & 1.98 & -1.51 & $0.33^{* *}$ & 7.76 & $3.72^{\star \star}$ & 0.19 \\
\hline VPL & 0.15 & 3.37 & -1.96 & -2.03 & 0 & 0.59 & 0.28 & -0.98 \\
\hline $\mathrm{cc}+\mathrm{ec}$ & $0.53^{\star * *}$ & 32.0 & $5.02^{\star \star \star}$ & $-4.63^{\star \star \star}$ & $0.48^{\star * \star}$ & 26.5 & $5.45^{\star \star \star}$ & $-3.17^{\star \star}$ \\
\hline $\mathrm{cc}+\mathrm{ec}+\mathrm{ic}$ & $0.51^{\star \star \star}$ & 44.2 & -1.09 & $-8.52^{\star \star \star}$ & $0.33^{\star \star \star}$ & 21.6 & $5.44^{\star \star \star}$ & $-5.23^{\star \star \star}$ \\
\hline $\mathrm{S} 1+\mathrm{VPL}$ & $0.39^{\star \star \star}$ & 18.6 & $-5.12^{\star \star \star}$ & -0.33 & $0.14^{\star}$ & 5.48 & $-2.95^{\star}$ & -0.52 \\
\hline
\end{tabular}

Statistically significant FDR-corrected $q$ values are shown ${ }^{*} q<0.05 ;{ }^{* *} q<0.01 ;{ }^{* \star *} q<0.001$ corresponding to uncorrected ${ }^{*} p<1.97 \times 10^{-2},{ }^{* *} p<2.5 \times 10^{-3}$, ${ }_{\star \star \star} p<1.22 \times 10^{-4}$, respectively; multiple linear regression test) for the Al, CD $\left(\times 10^{-2} \mathrm{cell} / \mu \mathrm{m}^{2}\right)$, and both parameters. AD, axial diffusivity; Al, anisotropy index; cc, corpus callosum; CD, cell density; ec, external capsule; FA, fractional anisotropy; ic, internal capsule; S1, somatosensory cortex; VPL, ventral posterolateral thalamic nucleus.

such as in the ipsilateral caudate putamen, hippocampus, ventrobasal complex, and cortical areas. In white matter areas, we also observed a volume reduction in the corpus callosum, cingulum, and internal capsule. After mTBI, a volume reduction in both white matter and gray matter areas is commonly observed in humans, and might indicate neural or axonal degeneration (Bigler, 2013; Mckee and Daneshvar, 2015). Until now, however, the few experimental studies that examined volumetric changes after mTBI using MRI volumetric analysis in rats revealed no brain volume changes (Kamnaksh et al., 2014; Wright et al., 2016). Kamnaksh et al. (2014), using DTI data in the mild blast injury model, reported no statistically significant volume changes in the hippocampus. On the contrary, we assessed local morphometric changes using T2-weighted images throughout the brain in the mild LFP injury model. Using the same animal model as in our study, Wright et al. (2016) did not find statistically significant total volume changes in the cortex, hippocampus, or corpus callosum using T2* data with ROI-based analysis; however, we reported local morphometric changes in the cortex and subcortical areas using T2-weighted deformationbased analysis.

In humans, the majority of studies that included MRI volumetric analysis performed after moderate and severe TBI reported volumetric abnormalities in the cortex, thalamus, putamen, and other regions (Gooijers et al., 2016; Cole et al., 2018). After mTBI, one human study also reported a volume reduction in cortical areas at one month after mTBI (Toth et al., 2013). Although Toth et al. (2013) observed a volume loss in cortical areas in the subacute phase of $\mathrm{mTBl}$, they observed increased volume in the cortical gray matter and decreased volume in the ventricles and extracerebral cerebrospinal fluid at $3 d$ post-mTBI. We demonstrated a volume reduction in white and gray matter areas, which progressed from the acute to the subacute phase of $\mathrm{mTBI}$, providing new insights in the detection of brain volume changes after $\mathrm{mTBI}$.

\section{Histology reveals axonal damage and gliosis that correlate with DTI after mTBI}

After the initial injury, an extensive complex cascade of molecular and cellular events occur, referred to as secondary injury (Jassam et al., 2017). Secondary cellular mechanisms include excitotoxicity and calcium flux, oxidative stress, mitochondrial dysfunction, and inflammation (Hill et al., 2016). This extensive secondary injury leads to cell death, impaired synaptic plasticity, and diffuse axonal injury, which compromise brain function (Walker and Tesco, 2013). Secondary damage after TBI has been assessed using DTI and histologic examination (Rodriguez-Paez et al., 2005; Mac Donald et al., 2007; Budde et al., 2011; Laitinen et al., 2015). To our knowledge, no other study has examined these changes using quantitative histologic analysis targeted on the basis of voxel-wise and morphologic MRI analyses. Previous studies demonstrated that decreased FA and AD parameters are widely associated with axonal injury after TBI (Mac Donald et al., 2007; Li et al., 2011; van de Looij et al., 2012). In the present study, we investigated the contribution of axonal damage and gliosis to the decreased FA and $A D$ in the subacute phase of $\mathrm{mTBI}$.

At $35 \mathrm{~d}$ post-mTBI, the most pronounced tissue changes were detected at the epicenter of the lesion, but we also observed alterations far from the primary lesion. 

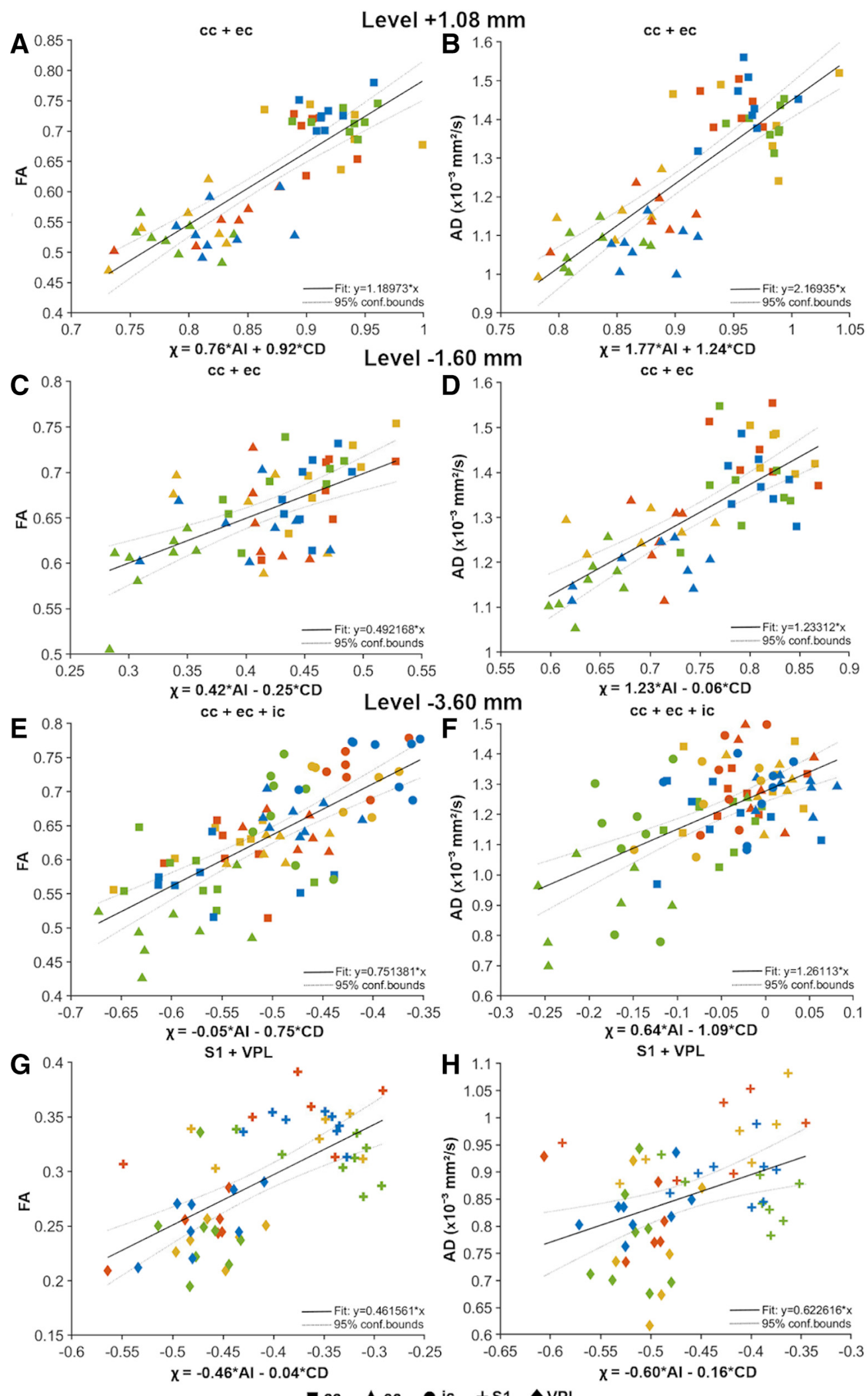

ac $\Delta$ ec ic $+\mathrm{S} 1 \bullet \mathrm{VPL}$

Ipsilateral Sham Contralateral Sham $\square$ Ipsilateral mTBI Contralateral mTBI

Figure 9. Representative multiple linear regression analyses of quantitative DTI and histologic analysis parameters at $+1.08 \mathrm{~mm}(\boldsymbol{A}$, $\boldsymbol{B}),-1.60 \mathrm{~mm}(\boldsymbol{C}, \boldsymbol{D})$, and $-3.60 \mathrm{~mm}(\boldsymbol{E}-\boldsymbol{H})$ from bregma. The thick black line is the regression line, and the two thin dotted lines represent the 95\% confidence interval. Both sham-operated and mTBI animals (ipsilateral and contralateral hemispheres) are represented by colors, and the brain areas by shapes. The $x$-axis represents the $\chi$ values obtained with the expression: $\chi=\beta$ Al $\times \mathrm{Al}+$ 
continued

$\beta_{\mathrm{CD}} \times \mathrm{CD}$, where $\beta$ is the weighting value. The $y$-axis represents $\mathrm{FA}$ or $\mathrm{AD}\left(\times 10^{-3} \mathrm{~mm}^{2} / \mathrm{s}\right)$. CD values are scaled $\left(\times 10^{-2}\right.$ cell/ $\left.\mu \mathrm{m}^{2}\right)$. $A D$, axial diffusivity; Al, anisotropy index; cc, corpus callosum; $C D$, cell density; ec, external capsule; FA, fractional anisotropy; ic, internal capsule; S1, somatosensory cortex; VPL, ventral posterolateral thalamic nucleus.

Specifically, rostral to the lesion site, we detected axonal damage and increased cellularity (gliosis) in both white and gray matter areas such as the corpus callosum, external capsule, and somatosensory cortex. The DTI results correlated with the histologic parameters in the white matter, but not in the gray matter. At the lesion site, a previous study using a mild LFP injury model reported axonal injury in white and gray matter areas such as the corpus callosum, internal and external capsule, cingulum, and thalamus on day 15 after mTBI (Hylin et al., 2013). That study, using both astrocyte and microglia markers, also demonstrated inflammation in the corpus callosum and thalamus. Nevertheless, our study demonstrated the presence of axonal damage and gliosis in white and gray matter regions such as the corpus callosum, external capsule, internal capsule, somatosensory cortex, and ventral posterolateral thalamic nucleus. This reveals the plausible contribution of both tissue components to the decreases in FA and AD after mTBI. Notably, we found more extensive tissue changes in the external capsule and somatosensory cortex compared with the above-mentioned areas in association with the loss of myelinated axons. No alterations in the DTI metrics were found in the somatosensory cortex by in vivo DTI, which might be related to the complexity of the structure. Similar to the somatosensory cortex, in vivo DTI did not detect alterations in the ventral posterolateral thalamic nucleus, although the histologic findings in this area revealed axonal damage and gliosis. Nevertheless, our multiple linear regression test showed a correlation between DTI and histology in both the white and gray matter, suggesting that both axonal damage and cellularity may contribute to the DTI metrics. Furthermore, the correlations showed a greater influence of the injury toward the primary lesion in the brain, consistent with our group analysis results. These findings together demonstrate that axonal injury and gliosis contribute to the alterations in DTI metrics in both the white and gray matter after mTBI.

\section{Technical limitations and future directions}

It is well known that the use of a simplistic tensor model in DTI underestimates the microstructure, especially in morphologically complex areas such as the gray matter (Tournier et al., 2011), which might explain the failure to detect mild microstructural changes in the somatosensory cortex and ventral posterolateral thalamic nucleus in this study. Therefore, future studies should focus on using more advanced diffusion MRI acquisitions and post-processing tools such as high-angular-resolution diffusion imaging, diffusion kurtosis, q-ball imaging, and neurite orientation dispersion and density imaging (Jensen et al., 2005; Kuo et al., 2008; Tournier et al., 2011; Zhang et al., 2012; Douglas et al., 2015). To quantitatively assess the DTI findings, we used ST analysis as a sensitive automated method offering anisotropy and orientation of histologic images equivalent to FA in DTI (Budde and Frank, 2012). In the present study, we used 2D histologic sections limiting the analysis to $2 \mathrm{D}$, and therefore, missing the full 3D histopathologic interpretation. Future studies should focus on using ST analysis of 3D histologic images acquired by advanced 3D microscopy methods such as confocal, multi-photon, or electron microscopy (Schilling et al., 2016; Salo et al., 2018; Abdollahzadeh et al., 2019).

It is important to highlight that this study uses only male rats and it may limit the translation of our findings into the clinic (Rubin and Lipton, 2019). Therefore, future preclinical studies are needed to assess the effect of sex on mTBI outcomes.

\section{Conclusion}

Our results indicate that in vivo DTI is sensitive for detecting widespread mild microstructural tissue alterations in white and gray matter areas throughout the brain in the acute phase of $\mathrm{mTBI}$. In the subacute phase of $\mathrm{mTBI}$, in vivo DTI detects progressive secondary tissue damage in the white and gray matter at the lesion site, but fails to detect changes distal to the primary lesion. We demonstrated that alterations in DTI metrics are associated with axonal damage and gliosis in both the white and gray matter. These findings offer new insights for imaging progressive tissue alterations after mTBI, highlighting the limitations of in vivo DTI for detecting mild microstructural damage, but opening up new ways to investigate tissue damage after $\mathrm{mTBI}$.

\section{References}

Abdollahzadeh A, Belevich I, Jokitalo E, Tohka J, Sierra A (2019) Automated 3D axonal morphometry of white matter. Sci Rep 9:6084.

Amyot F, Arciniegas DB, Brazaitis MP, Curley KC, Diaz-Arrastia R, Gandjbakhche A, Herscovitch P, Hinds SR, Manley GT, Pacifico A, Razumovsky A, Riley J, Salzer W, Shih R, Smirniotopoulos JG, Stocker D (2015) A Review of the effectiveness of neuroimaging modalities for the detection of traumatic brain injury. $J$ Neurotrauma 32:1693-1721.

Andersson JLR, Sotiropoulos SN (2016) An integrated approach to correction for off-resonance effects and subject movement in diffusion MR imaging. Neuroimage 125:1063-1078.

Arciniegas DB, Anderson CA, Topkoff J, McAllister TW (2005) Mild traumatic brain injury: a neuropsychiatric approach to diagnosis, evaluation, and treatment. Neuropsychiatr Dis Treat 1:311-327.

Arfanakis K, Haughton VM, Carew JD, Rogers BP, Dempsey RJ, Meyerand ME (2002) Diffusion tensor MR imaging in diffuse axonal injury. Am Soc Neuroradiol 23:794-802.

Avants BB, Epstein CL, Grossman M, Gee JC (2008) Symmetric diffeomorphic image registration with cross-correlation: evaluating automated labeling of elderly and neurodegenerative brain. Med Image Anal 12:26-41.

Avants BB, Tustison NJ, Song G, Cook PA, Klein A, Gee JC (2011) A reproducible evaluation of ANTs similarity metric performance in brain image registration. Neuroimage 54:2033-2044. 
Basser PJ, Mattiello J, LeBihan D (1994) MR diffusion tensor spectroscopy and imaging. Biophys J 66:259-267.

Benjamini Y, Hochberg Y (1995) Controlling the false discovery rate: a practical and powerful approach to multiple testing. R Stat Soc 57:289-300.

Bigler ED (2013) Traumatic brain injury, neuroimaging, and neurodegeneration. Front Hum Neurosci 7:1-15.

Budde MD, Frank JA (2012) Examining brain microstructure using structure tensor analysis of histological sections. Neuroimage 63:1-10.

Budde MD, Janes L, Gold E, Turtzo LC, Frank JA (2011) The contribution of gliosis to diffusion tensor anisotropy and tractography following traumatic brain injury: validation in the rat using Fourier analysis of stained tissue sections. Brain 134:2248-2260.

Budde MD, Shah A, McCrea M, Cullinan WE, Pintar FA, Stemper BD (2013) Primary blast traumatic brain injury in the rat: relating diffusion tensor imaging and behavior. Front Neurol 4:1-12.

Chan TF, Vese LA (2001) Active contours without edges. IEEE Trans Image Process 10:266-277.

Cole JH, Jolly A, De Simoni S, Bourke N, Patel MC, Scott G, Sharp DJ (2018) Spatial patterns of progressive brain volume loss after moderate-severe traumatic brain injury. Brain 141:822-836.

Douglas DB, Iv M, Douglas PK, Anderson A, Vos SB, Bammer R, Zeineh M, Wintermark M (2015) Diffusion tensor imaging of TBI: potentials and challenges. Top Magn Reson Imaging 24:241-251.

Gardner RC, Yaffe K (2015) Epidemiology of mild traumatic brain injury and neurodegenerative disease. Mol Cell Neurosci 66:75-80.

Gaser C, Nenadic I, Buchsbaum BR, Hazlett EA, Buchsbaum MS (2001) Deformation-based morphometry and its relation to conventional volumetry of brain lateral ventricles in MRI. Neuroimage 13:1140-1145.

Gooijers J, Chalavi S, Beeckmans K, Michiels K, Lafosse C, Sunaert S, Swinnen SP (2016) Subcortical volume loss in the thalamus, putamen, and pallidum, induced by traumatic brain injury, is associated with motor performance deficits. Neurorehabil Neural Repair 30:603-614.

Grandhi R, Tavakoli S, Ortega C, Simmonds MJ (2017) A review of chronic pain and cognitive, mood, and motor dysfunction following mild traumatic brain injury: complex, comorbid, and/or overlapping conditions? Brain Sci 7:1-8.

Harris TC, De Rooij R, Kuhl E (2019) The shrinking brain: cerebral atrophy following traumatic brain injury. Ann Biomed Eng 47:19411959.

Herrera JJ, Bockhorst K, Kondraganti S, Stertz L, Quevedo J, Narayana PA (2017) Acute white matter tract damage after frontal mild traumatic brain injury. J Neurotrauma 34:291-299.

Hill CS, Coleman MP, Menon DK (2016) Traumatic axonal injury: mechanisms and translational opportunities. Trends Neurosci 39:311-324.

Hutchinson EB, Schwerin SC, Avram AV, Juliano SL, Pierpaoli C (2018) Diffusion MRI and the detection of alterations following traumatic brain injury. J Neurosci Res 96:612-625.

Hylin MJ, Orsi SA, Zhao J, Bockhorst K, Perez A, Moore AN, Dash PK (2013) Behavioral and histopathological alterations resulting from mild fluid percussion injury. J Neurotrauma 30:702-715.

Inglese M, Makani S, Johnson G, Cohen BA, Silver JA, Gonen O, Grossman RI (2005) Diffuse axonal injury in mild traumatic brain injury: a diffusion tensor imaging study. J Neurosurg 103:298-303.

Jassam YN, Izzy S, Whalen M, McGavern DB, El Khoury J (2017) Neuroimmunology of traumatic brain injury: time for a paradigm shift. Neuron 95:1246-1265.

Jensen JH, Helpern JA, Ramani A, Lu H, Kaczynski K (2005) Diffusional kurtosis imaging: the quantification of non-Gaussian water diffusion by means of magnetic resonance imaging. Magn Reson Med 53:1432-1440.

Johnson VE, Stewart W, Smith DH (2013) Axonal pathology in traumatic brain injury. Exp Neurol 246:35-43.

Kamnaksh A, Budde MD, Kovesdi E, Long JB, Frank JA, Agoston DV (2014) Diffusion tensor imaging reveals acute subcortical changes after mild blast-induced traumatic brain injury. Sci Rep 4:4809.
Kharatishvili I, Nissinen JP, McIntosh TK, Pitkänen A (2006) A model of posttraumatic epilepsy induced by lateral fluid-percussion brain injury in rats. Neuroscience 140:685-697.

Kim JJ, Gean AD (2011) Imaging for the diagnosis and management of traumatic brain injury. Neurotherapeutics 8:39-53.

Kinnunen KM, Greenwood R, Powell JH, Leech R, Hawkins PC, Bonnelle V, Patel MC, Counsell SJ, Sharp DJ (2011) White matter damage and cognitive impairment after traumatic brain injury. Brain 134:449-463.

Kuo LW, Chen JH, Wedeen VJ, Tseng WYI (2008) Optimization of diffusion spectrum imaging and q-ball imaging on clinical MRI system. Neuroimage 41:7-18.

Laitinen T, Sierra A, Pitkänen A, Gröhn O (2010) Diffusion tensor MRI of axonal plasticity in the rat hippocampus. Neuroimage 51:521530.

Laitinen T, Sierra López A, Bolkvadze T, Pitkänen A, Gröhn O (2015) Diffusion tensor imaging detects chronic microstructural changes in white and grey matter after traumatic brain injury in rat. Front Neurosci 9:1-12.

Li J, Li XY, Feng DF, Gu L (2011) Quantitative evaluation of microscopic injury with diffusion tensor imaging in a rat model of diffuse axonal injury. Eur J Neurosci 33:933-945.

Li W, Long JA, Watts L, Shen Q, Liu Y, Jiang Z, Duong TQ (2016) Spatiotemporal changes in diffusion, T2 and susceptibility of white matter following mild traumati brain injury. NMR Biomed 29:896903.

Long JA, Watts LT, Chemello J, Huang S, Shen Q, Duong TQ (2015) Multiparametric and longitudinal MRI characterization of mild traumatic brain injury in rats. J Neurotrauma 32:598-607.

Mac Donald CL, Dikranian K, Song SK, Bayly PV, Holtzman DM, Brody DL (2007) Detection of traumatic axonal injury with diffusion tensor imaging in a mouse model of traumatic brain injury. Exp Neurol 205:116-131.

Marion CM, Radomski KL, Cramer NP, Galdzicki Z, Armstrong RC (2018) Experimental traumatic brain injury identifies distinct early and late phase axonal conduction deficits of white matter pathophysiology, and reveals intervening recovery. J Neurosci 38:87238736.

Mayer AR, Quinn DK, Master CL (2017) The spectrum of mild traumatic brain injury. Neurology 89:623-632.

Mclnnes K, Friesen CL, MacKenzie DE, Westwood DA, Boe SG (2017) Mild traumatic brain injury (mTBI) and chronic cognitive impairment: a scoping review. PLoS One 12:e0174847.

Mckee AC, Daneshvar DH (2015) The neuropathology of traumatic brain injury. Handb Clin Neurol 127:45-66.

Messé A, Caplain S, Paradot G, Garrigue D, Mineo JF, Soto Ares G, Ducreux D, Vignaud $F$, Rozec $G$, Desal $H$, Pélégrini-Issac $M$, Montreuil M, Benali H, Lehéricy S (2011) Diffusion tensor imaging and white matter lesions at the subacute stage in mild traumatic brain injury with persistent neurobehavioral impairment. Hum Brain Mapp 32:999-1011.

Mori S, Zhang J (2006) Principles of diffusion tensor imaging and its applications to basic neuroscience research. Neuron 51:527-539.

Mutch CA, Talbott JF, Gean A (2016) Imaging evaluation of acute traumatic brain injury. Neurosurg Clin N Am 27:409-439.

Noble S, Scheinost D, Constable RT (2020) Cluster failure or power failure? evaluating sensitivity in cluster-level inference. Neuroimage 209:116468.

Pajevic S, Pierpaoli C (1999) Color schemes to represent the orientation of anisotropic tissues from diffusion tensor data: application to white matter fiber tract mapping in the human brain. Magn Reson Med 42:526-540.

Pierpaoli C, Jezzard P, Basser PJ, Barnett A, Di Chiro G (1996) Diffusion tensor imaging of the human brain. Radiology 201:637648.

Rodriguez-Paez AC, Brunschwig JP, Bramlett HM (2005) Light and electron microscopic assessment of progressive atrophy following moderate traumatic brain injury in the rat. Acta Neuropathol 109:603-616. 
Rubin TG, Lipton ML (2019) Sex differences in animal models of traumatic brain injury. J Exp Neurosci 13:1179069519844020.

Rutgers DR, Toulgoat F, Cazejust J, Fillard P, Lasjaunias P, Ducreux D (2008) White matter abnormalities in mild traumatic brain injury: a diffusion tensor imaging study. AJNR Am J Neuroradiol 29:514519.

Salo RA, Belevich I, Manninen E, Jokitalo E, Gröhn O, Sierra A (2018) Quantification of anisotropy and orientation in 3D electron microscopy and diffusion tensor imaging in injured rat brain. Neuroimage 172:404-414.

Schilling K, Janve V, Gao Y, Stepniewska I, Landman BA, Anderson AW (2016) Comparison of 3D orientation distribution functions measured with confocal microscopy and diffusion MRI. Neuroimage 129:185-197.

Shenton ME, Hamoda HM, Schneiderman JS, Bouix S, Pasternak O, Rathi Y, Vu M-A, Purohit MP, Helmer K, Koerte I, Lin AP, Westin CF, Kikinis R, Kubicki M, Stern RA, Zafonte R (2012) A review of magnetic resonance imaging and diffusion tensor imaging findings in mild traumatic brain injury. Brain Imaging Behav 6:137-192.

Sidaros A, Engberg AW, Sidaros K, Liptrot MG, Herning M, Petersen P, Paulson OB, Jernigan TL, Rostrup E (2008) Diffusion tensor imaging during recovery from severe traumatic brain injury and relation to clinical outcome: a longitudinal study. Brain 131:559-572.

Sierra A, Laitinen T, Gröhn O, Pitkänen A (2015) Diffusion tensor imaging of hippocampal network plasticity. Brain Struct Funct 220:781-801.

Singh K, Trivedi R, Devi MM, Tripathi RP, Khushu S (2016) Longitudinal changes in the DTI measures, anti-GFAP expression and levels of serum inflammatory cytokines following mild traumatic brain injury. Exp Neurol 275:427-435.

Smith SM, Nichols TE (2009) Threshold-free cluster enhancement: addressing problems of smoothing, threshold dependence and localisation in cluster inference. Neuroimage 44:83-98.

Stokum JA, Gerzanich V, Simard JM (2016) Molecular pathophysiology of cerebral edema. J Cereb Blood Flow Metab 36:513-538.

Tae WS, Ham BJ, Pyun SB, Kang SH, Kim BJ (2018) Current clinical applications of diffusion-tensor imaging in neurological disorders. J Clin Neurol 14:129-140.
Toth A, Kovacs N, Perlaki G, Orsi G, Aradi M, Komaromy H, Ezer E, Bukovics P, Farkas O, Janszky J, Doczi T, Buki A, Schwarcz A (2013) Multi-modal magnetic resonance imaging in the acute and sub-acute phase of mild traumatic brain injury: can we see the difference? J Neurotrauma 30:2-10.

Tournier JD, Mori S, Leemans A (2011) Diffusion tensor imaging and beyond. Magn Reson Med 65:1532-1556.

Tu TW, Lescher JD, Williams RA, Jikaria N, Turtzo LC, Frank JA (2017) Abnormal injury response in spontaneous mild ventriculomegaly Wistar rat brains: a pathological correlation study of diffusion tensor and magnetization transfer imaging in mild traumatic brain injury. J Neurotrauma 34:248-256.

van de Looij Y, Mauconduit F, Beaumont M, Valable S, Farion R, Francony G, Payen JF, Lahrech H (2012) Diffusion tensor imaging of diffuse axonal injury in a rat brain trauma model. NMR Biomed 25:93-103.

Walker KR, Tesco G (2013) Molecular mechanisms of cognitive dysfunction following traumatic brain injury. Front Aging Neurosci 5:125.

Westin CF, Maier SE, Mamata H, Nabavi A, Jolesz FA, Kikinis R (2002) Processing and visualization for diffusion tensor MRI. Med Image Anal 6:93-108.

Winkler AM, Ridgway GR, Webster MA, Smith SM, Nichols TE (2014) Permutation inference for the general linear model. Neuroimage 92:381-397.

Wright DK, Trezise J, Kamnaksh A, Bekdash R, Johnston LA, Ordidge R, Semple BD, Gardner AJ, Stanwell P, O'Brien TJ, Agoston DV, Shultz SR (2016) Behavioral, blood, and magnetic resonance imaging biomarkers of experimental mild traumatic brain injury. Sci Rep 6:28713.

Yekutieli D, Benjamini Y (1999) Resampling-based false discovery rate controlling multiple test procedures for correlated test statistics. J Stat Plan Inference 82:171-196.

Zhang H, Schneider T, Wheeler-Kingshott CA, Alexander DC (2012) NODDI: practical in vivo neurite orientation dispersion and density imaging of the human brain. Neuroimage 61:1000-1016.

Zhuo J, Xu S, Proctor JL, Mullins RJ, Simon JZ, Fiskum G, Gullapalli RP (2012) Diffusion kurtosis as an in vivo imaging marker for reactive astrogliosis in traumatic brain injury. Neuroimage 59:467-477. 